\title{
ZUR LAU'TVERSCHIEBUNG.
}

Der hauptzweck der nachfolgenden untersuchung ist die bbestimmung des ganges, welchen die verschiebung der indogernmanischen aspiraten im germanischen genommen hat. Dazu sisind drei fragen zu beantworten; erstens: welches war die ursppriingliche gestalt der aspiraten in der indogermanischen urspprache; zweitens: welches die in der germanischen ursprache: ddrittens: durch welche etwaige zwischenglieder war der ubberggang von der einen in die andere vermittelt. Ohne die richtige bbeantwortung der beiden ersten fragen fehlt natirlich allen specculationen uber die dritte der bodun. Die erste ist der gegensstand vielfältiger erörterungen gewesen. Noch immer stehen ssich sehr abweichende ansichten gegentiber. Aber doch halte iuch dafür, worauf ich später zurückkomme, dass wir zu einem bbestimmten ron den meisten hervorragenden sprachforschern aanerkannten resultate gelangt sind. Die zweite frage aber ist eaigentlich noch gar nicht aufgeworfen. Man hat sich stets begrnuigt vom gotischen auszugehen, und hat dabei meist olme wveiteres die gotischen laute denjenigen gleicligesetzt, welche die leateinischen buchstaben ausdrücken, durch welche sie in der grewöhnlichen umschreibung bezeichnet werden. Bei scherer errscheint zwar zum teil eine andere auffassung; aber sie ist mur schwankend ausgenprochen und nicht consequent durchgeftuhrt. Nun ist aber ein unbedingtes erfordernis zur bestimmung dler urgermanischen aussprache dieser latute eine vergleichende bvetrachtung der gestaltungen derselben in sämmtlichen germamischen sprachen, und zwar eine solche, welche sich nicht bloss an den buchstaben hält, sondern, soweit dazu mittel vorhanden siind, den lautwert festzustellen sucht. Erst mit hulfe dieser fieststellung kann sowol die weiterverschiebung in den eiuzelnen mundarten, als das verhältnis zu den verwanten sprachfamilien 
in das rechte licht gesetzt werden. Ich will also zunäichst cinen versuch dazu machen. Die frage nach aussprache und geschichte der den indogermanischen aspiraten entsprechenden sogenannten deutschen medien $b, g, d$ hängt überall aufs engste zusammen mit der untersuchung iiber die indogermanischer tenuis enteprechenden laute $f, h, f$, die wir daher in mecre betrachtumy einschliessen müssen.

Ueber die aussprache des gotischen besitzen wir awei monographien, von Weingärtner, Leipzig 1858 und ron Dietrich, Marburg 1862. Die ansichten dieser beiden gelehrten wehen wie über die ausprache der vokale, so auch iiber die der hier in betracht kommenden consonanten riemlich weit anseinander. Eine neue priffung ist daher nötig. Die kriterien, welche uns zu gebote stehen, sind einr rseits das verhältnis der gotischen zu den griechischen und lateinischen lautzeichen, anderseits gewisse lautgesetzliche veränderungen. Was die ausprarche des griechischen zur zeit des Ulfilas angeht, so steht es fest, dass die medien $\beta, \gamma, \delta$ bereits als tönende, die aspiraten $\mathscr{f}, \mathcal{g}$ als tonlose spiranten gesprochen wurden; weniger sicher ist das letztere für $\chi$, was wir iibrigens für unsern zweck ganz bei seite lassen können. Zu beachten ist noch, dass $\beta$ und q als rein labiale, nicht labiodentale spiranten aufzufassen sincl. Nun sind die gotischen zeichen für $b, g, d$ entstanden aus den griechischen $\beta, \gamma, \delta$; es ist also das nächstlicgende ihren lautwert denselben gleich zu setzen, so lange nichts anderes dagegen spricht. Hinwider wird gotisches $f$ und $f$ nicht durch $q$ und $\vartheta$ bezeichnet, sondern durch einheimische runenzeichen; das erweckt den verdacht, dass die aussprache etwas verschiedcn gewesen sei, ohne dass indessen darauf ein hstimmter beweis gegrtindet werden könnte, da die beibehaltungr der runen auch auf anderen grinden beruhen kann. Entscheidender ist die widergabe der gricehisehen und lateinischen wörter im gotischen, und umgekehrt die der gotischen namen bei griechischen und lateinischen schriftstellorn. Es werden num nicht bloss die gricchischen medien durch die entsprechenden gotischen widergegeben, sondern auch $\varphi$ durch $f$, it durch $p ; h$ entspricht dem spiritus asper; über seine natur ist uiberhaupt kein streit mehr. Fur die aussprache der medien als spiranten spricht norh, dass in einem falle $d=\vartheta$ ist, baidsaiidan Luc. 9, 10, und mehrmals 
$b=\mathscr{f}$ in Asabis Esdr. 2, 41 und Joseba. Dagegen kann es nicht sehr ins gewicht fallen, dass zweimal in Lod $d$ einen $\tau$ und einmal in falaiy $g$ einem $x$ entspricht, so wenig wie man aus dem einmaligen peimaupaius tür Truótros auf eine von der des i rersehicdene anssprathe des $f$ schliessen wird. Es können hicr alweichungen des griechischen textes oder Hüchtigkeiten des iibersetzers vorliegen. Die vertretung des lateinischen v durch $b$ in silbarus, naubaimbair beweist noch bestimmter die spirantische aussprache des letzteren wenigstens im inlaut. Lateinisch'm b entspricht es nur in anakumbjan und naubaimbair wol zu beachten hach $m$, in welcher stellung, wie wir sehen werden, auch in den iibrigen dialekten die aussprache abweicht; doch ist diess an und für sich kein zwingender beweis für die völiige gleichheit des lateinischen und gotisehen $b$, da eben kein anderes zeichen zum ausdruck des ersteren zu gebote stand und da das wort bereits eingeluurgert zu sein scheint. Die widergabe der gotischen eigemnamen hei den griechischen und lateinischen schriftstellern ist besonders ausführlich ron Dietrich besprochen. Ich kann aber den schlissen, die derselbe daraus anf die aussurache zicht, nicht durehgängig beistimmen. Man darf iibrigens bei der betrachtung derselben nicht aus dem auge verlieren, dass die gotischen lante wie die anderer dialekte in einem stïn fortschreiten hegriffen gewesen sind, so dass die s.mache olune ihr fribes aussterben aller wahrscheinlichkeit nach bis $z$ derselben stufe wie das strengalthochdeutsche gelangt sein wiirle. Wir diirfen daher die lautgestaltungen in späteren quellen nicht ohne weiteres auf die zeit des Ulfilas ubertragen. Am meisten klären uns die lateinisehen namensformen über die ausspraclue des $b$ aut. Diess wird im inlaut zwischen rokalen regelmässig durch $v$ willergegreben (Dictr. s. 71), auch nach $l$ kimmt $v$ vor. Wenn sonst im inlatut in consonantenverbinduggen und im anlaut $b$ dafür steht, so beweist diess noch nicht sither, dass das gotische $b$ hier als explosivlaut gesprochen wurde. War es wie $\beta$ ein rein labialer spirant,- so entsprach ihm weder das lateinische labiodentale $v$ noch das $b$ rollkomnen, sondern der laut hatte etwas mittleres zwischen beiden, wodurch ein schwanken in der schreibung veranlasst werden kunnte. $b$ wird ja auch mit vorgesetztem $u$ für deutsches $w$ żbraucht (Dictr. s. 79) z.b. Ubadila, wo es nur den rein labi- 
alen spiranten bezeichnen kann. Doch die consequente setzung von $b$ im anlaut neben $v$ im inlaut scheint eine verschiedenheit der aussprache anzudeuten. Nur im zweiten teile eines compositums, dessen anlaut sonst wie der eines selbständigen wortes betrachtet wird, findet sich $v$ in Reciverga. Wenn gotischem $g$ und $d$ lateinisches $g$ und $d$ entspricht, so werden wir widerum daraus nicht mit notwendigkeit schliessen, dass die aussprache gleich war. Man könnte sonst mit demselben rechte schliessen, dass auch $\gamma$ und $\delta$ in der späteren zeit als verschlusslaute gesprochen wären. Die gotischen laute konnten mur lurch die zeichen ausgedrückt werden, deren lautwert ihnen am nächsten kam. Die spirantische natur des $g$ im inlaut ist auch von Dietrich zugegeben und durch zahlreiche beispiele des rerklingeas von $g$ im inlaut und auch im anlaut des zweiten teils der composita wahrscheinlich gemacht. Wenn aber die widergabe durch lateinisches $g$ nicht als ein beweis gegen die aussprache des gotischen $g$ als spirant angesehen wird, so darf uns auch lateinisches für gotisches $d$ nicht stören. Im anlaut wird gotisches $g$ ofter durch $c$ widergegeben, schon bei Ammianus. Wir mutssen hierin gewis einen beweis dafur finden, dass hier kurz nach Ulfilas ein versehlusslaut gesprochen wurde. Gotisches $\boldsymbol{b}$ wird durch $t h$ vertreten, also durch dasselbe zeichen wie $\vartheta$, dem wir demnach die gleiche bedeutung zuzuschreiben berechtigt sind. Wenn dafür häufig einfaches $t$ steht, so ist diess wol blosse schreibernachlässigkeit, der wir ebenso später bei der schreibung deutscher eigennamen in lateinischen büchern und urkunden begegnen. $d$ fur $b$ im inlaut beruht auf weiterer fortbewegung des lautes. Gotisches $f$ wird seltener durch $f$ widergegeben als durch $p h$. Daraus folgt nicht, dass es cinen sehr harten klang gehabt hat, wie Dietrich sagt, sondern nur, dass es rein labial war wie $\varphi$, weshallb es im lateinischen durch dasselbe zeichen wie dieses umschrieben werden muste. $p$ für gotisches $f$ ist jedenfalls aufzufassen wie $t$ für $b$. Es mag auch sein, dass beide nicht bloss auf nachlässiger schreibung beruhen, sondern auch auf ungenauer auffassung mit dem ohre. Bei hastiger aussprache kann die spirans wenigstens ebenso gut als tenuis aufgefasst werden, wie die aspirata oder affiricata, von welchen letzteren es übrigens gar nicht sicher ist, ob sie wirklich die mittelstufen zwischen 
incdogermanischer tenuis und germanischer spirans gebildet harben.

Fassen wir zusammen, was sich von dieser seite für die ausssprache der medien ergibt, so lïsst sich allerdings nicht in abmrede stellen, dass immer einige schwierigkeiten und widersprrüche bleibcn, die nicht bis zur zweifellosigkeit gelöst werden köinnen. Indessen wenn man die unausführbarkeit ciner vollkommen genauen umschreibung in ein fremdes alphaket erwägt, so werden einzehe kleine bedenken in rucksicht hierauf schwinde'n müssen, und man muss das resultat anerkennen, dass sehr gewichtige gründe vorhanden sind die medien im inlaut nach rokalen als spiranten aufzufassen, während anderseits die ausspirache im anlaut und im inlaut nach liquida und vor allem nach nasal daron verschieden gewesen sein muss, sodass hier entweder verschlusslaute gesprochen wurden oder, wofür die gleiche schreibung und das verhältnis zum griechischen spricht, iibergangslaute zwischen verschluss - und reibelauten.

Entscheidender als alles bisher vorgebrachte ist die beobarchtung des lautwandels im gotischen. Es ist bekannt, dass in denselben wörtern, welche im inlaut $b$ und $d$ zeigen, im auslaut und vor dem nominativzeichen $s$ statt dessen meistens $f$ und $p$ erscheint, ausgenommen nach consonanten $m, n, l, r, z$. Wo wir sonst einen solchen wechsel zwischen in - und auslaut in den germanischen sprachen finden, berult derselbe darauf, lass im inlaut ein tönender, im auslaut der entsprechende tonlose laut gesprochen wird. So spricht man in Oberdeutschland tak und liep fïr lay und liel, in Niederdeutschland dach und leif, weil hier im inlaut tönende spiranten gesprochen werden: dughe, leiwe. Dasselbe verhältnis sind wir berechtigt für das gotische anzumehmen. Da $f$ anerkanntermassen reine npirans ist, so muss $b$ die ilm entsprechende tönende spirans gewesen scin. Wäre ferner $p$ aspirata, $d$ tönender verschlusslaut gewesen, so wäre gar nicht abzuschen, wie sie dazu kämen mit einander zu wechseln. Sic können sich vielmehr nur durch vorhaudensein oder fehlen des stimmtones unterschieden haben, und wir müssen ihren lautwert gleichfalls als tönende und tonlose spirans bestimmen. Auf diess verhältnis hat schon Weingärtner bedeutendes gewicht gelegt. Wenn im auslaut $f$ und $f$ nicht ganz consequent geschrieben werden, sondern daneben 
$b$ und $d$, so rührt diess nur daher, dass nicht uberall streng phonetisch geschrieben wurde, wie diess im neuhochdeutschen in diesem falle gar nicht geschieht. Dass nach consonanten die media bleibt, beweist wider eine abweichende aussprache. Da aber in diesen fällen auch nicht die tenuis geschrieben wird, so ist es doch nicht sicher, ob hier wirklicher verschlusslaut gesprochen wurde. Wenn zwischen $g$ und $h$ nicht derselbe regelmässige wechsel besteht, so kann daraus nicht gefolgert werden, lass $g$ nicht spirant gewesen wäre, sondern da $h$ bereits zum blossen hauche abgeschwächt war, so war es nicht geschickt dcu dem $g$ entsprechenden harten spiranten $=$ mhd. ch auszudricken, und es konnte passender in ermanglung eines bessern das zeichen für den weichen spiranten beibehalten werden.*) Es wäre allenfalls noch denkbar, dass $f$ und $f$ als harte affiricaten aufzufassen wären, und damn $b$ und $d$ als die entsprechenden weichen, wie es Scherer für möglich hält. Aber das widerspricht durchau: dem, was wir vorher über die natur der laute ersehlossen haben. Wie hätte z. b. $b v$ durch einfaches lateinisches $v$ widergegeben werden sollen? Man hat tiberhaupt keine andere reranlassung medienafficaten im gotischen anzunehmen, als die, wie wir sehen werden, unbegrundete voraussetzung, dass wol aus einer solchen, aber nicht aus einer einfachen spirans sich eine mit verschluss gebildete media entwickeln könnte. Den $b$ eine andere natur zuzuschreiben als dem $f$ berechtigt nichts; ihr beiderseitiges verhältnis ist röllig analog.

Wir haben bisher diesen lautwechsel nur rom standpunkte des gotischen aus betrachtet. Von diesem aus gesehen, scheint es, dass uberall der weiche laut der ältere ist, der nur im auslaut fast mit naturnotwendigkeit in den harten ubergeht. Die vergleichung der verwanten sprachen aber eryibt, dass in don meisten fällen der harte laut der :iltere ist, keineswegs aber durchgängig. Fälle, in denen die gotische media einer indogermanischen aspirata entspricht, also zunächst weich sein muss, sind baup (praet. von biudan), juggalaups, rairop (praet. von redan); von liubs, galaubs, stabs, rauds kommen zufällig nur formen vor,

*) Bei Jordanes findet sich Berich neben Berig (Dietrich s. 75), was also dem iibergang von $b$ in $f$ und $d$ in $p$ analog sein wiirde. 
in denen $b$ und $d$ inlautend zwischen vokalen steht; es könnte daher eben so gut liufs, raujs etc. angesetzt werden. Eine rerhărtung im auslaut findet also sicher statt. In den meisten wörtern, in denen der wechsel zwischen $b$ und $f, d$ und $b$ sich zeigt, entsprechen diese laute indogermanischer tenuis. Auch sonst sind die medien $b, d$ und auch $g$ aus ursprunglicher tenuis hervorgegangen. Die hierher gehörigen wörter sind am vollständigsten zusammengestellt in Lottners abhandlung „uber die ausnahmen der ersten lautverschiebung " Kuhns zeitschr. XI, 161 ff. auf seite $187 \mathrm{ff}$. Es ist wol kaum noch nötig die früher verbreitete ansicht zu widerlegen, dass diese medien unmittelbar aus der tenuis erweicht seien. Scherer hat eine widerlegung derselben gegeben in der zeitschr. f. d. östr. gymn. XII, 648. 49. Er stellt drei möglichkeiten auf: entweder die erweichung hat stattgefunden vor der lautverschiebung, oder nach der verschiebung, oder sie fällt zwischen die einzelnen akte der verschiebung. Fiele sie vor die verschiebung, so hätte die aus der tenuis erweichte media durch dieselbe wider zur tenuis werden müssen. Zwischen die verschiebung könnte sie nach Scherer fallen, wenn man dieselbe reihenfolge wie Curtius annimmt, nämlich zwischen dessen zweiten und dritten akt, der verschiebung der media zur tenuis und der der media zur aspirata. Ich möchte auch diese möglichkeit bestreiten. Denn dann wäre es wenigstens ein sehr wunderbarer zufall, dass nicht auch eine anzahl aus der media entstandener tenues erweicht sind. Und überhaupt wäre es unwahrscheinlich, dass unmittelbar nach der eben vollzogenen durchgängigen verhärtung gleich wider eine ausgedehnte orweichung gefolgt wäre. Die erweichung muss also nach der verschiebung der indogermanischen media und der der tenuis stattgefunden haben. Der voll der erweichung betroffene laut war also nicht mehr die tenuis, sondern eine durch die verschiebung bewirkte modification derselben. Es fragt sich nur, bis zu welcher stufe die verschiebung gediehen war. Um diese frage zu entscheiden, wäre zun̈chst zu bestimmen, auf welche weise die germanische verschiebung der tenuis vor sich geyangen sein kann. Entweder wurde, wie die gewöhnliche annahme ist, aus der tenuis zunichst die aspirata, aus dieser daun ein doppellaut bestehend ats tenuis und homorganer spiraus, den man affricata zu nennen Jeiträge zur geschichte der deutschen sprache. I. 
gewohnt ist, und daraus endlich einfache spirans, oder es wurde aus der tenuis unmittelbar die affricata, wio der vorgang vielleicht im hochdeutschen war, und daraus wider die spirans, oder endlich drittens, eine ansicht, die von Scherer vertreten wird, (z. gesch. 82, z. f. d. östr. gymn. XII, 656) es wurde unmittelbar aus der tenuis die spirans. Alles dreies ist physiologisch vallkommen möglich. Die letzte annahme setzt, wie Scherer richtig bemerkt, keinen sprung voraus. Der vorgang ist einfach der, dass bei sonst gleicher mundstellung der verschluss gelockert wird. Unmittelbaren thbergang von tonlosem verschlusslaut in einen reibelaut weiss ich allerdings sonst nicht mit sicherheit nachzuweisen, sehr häufig ist aber der vollkommen analoge von tönendem verschlusslaut in reibelaut, z. b. im griechischen und in den romanischen sprachen. Scherer beruft sich furr seine ansicht besonders darauf, dass das resultat der germanischen verschiebung einfache spiranten sind, während im hochdeutschen im anlaut affricata erscheint.*) Es ist richtig, dass durch diesen umstand seine annahme uberhaupt möglich wird, aber erwiesen ist sie damit nicht. Die wandelung durch die aspirata hindurch brauchte nicht bis zur einfachen spirans fortzuschreiten, wie das althochdeutsche beweist, konnte es aber sehr wol, wofur das griechische und lateinische die sichern belege gibt. Wir mussen also die frage einstweilen unentschieden lassen. Wurde die tenuis unmittelbar in die spirans verwandelt, so ist ohne weiteres klar, dass in den fraglichen fällen $g, d, b$ durch erweichung aus der harten spirans entstanden sein mlissen. Liegen dagegen dio erwähnten ubergangsstufen dazwischen, so kann es in frage kommen, ob nicht bereits auf einer von diesen die orweichung eingetreten ist; ob also vielleicht aspirata oder affricata davon betrofien wurde. Zunächst scheint mir die verwandlung von wirklicher harter aspirata $=$ tenuis $+\mathrm{h}$ in die weiche $=$ media $+\mathrm{h}$ eine physiologivehe unmöglichkeit. Es ist bekannt, wie sehr sich unser bedeutendster sprachphysiologe, Brucke, gegen die anerkennung der sprech-

-) Auch fuir das hochdeutsche hat Scherer im inlaut nach vokalen unmittelbaren tibergang in die spirans angenommen. Diese ansicht ist viderlegt und der durchgang durch die afficata nachgewiesen von Braume in diesem heft $\mathrm{s}$. $48 \mathrm{ff}$. 
barkeit der medialaspiraten gesträubt hat. Wenn nun auch durch die bemerkungen von Arendt in den beiträgen z. vergl. sprachf. II, $283 \mathrm{ff}$. die existenz derselben ausser zweifel gesetzt ist, so sind sie doch immer sehr schwierige lautverbindungen deren sich deshalb die meisten sprachen entledigt haben, und es ist gar nicht denkbar, dass sie aus der gar keine schwierigkeit bietenden verbindung tenuis $+h$ sollten entstanden sein. Die verwandlung von tenuisaffricata in medienaffricata wäre mindestens unwahrscheinlich. Nirgends findet sich ein analogon dazu, wie denn überhaupt die medienaffricaten nirgends in einer sprache nachgewiesen, sondern nur erschlossen sind. So schwere consonantenverbindungen sind nicht leicht dergleichen veränderungen ausgesetzt. Ueberdiess würde die erweichung derselben eine erweichung der tenuis in sich schliessen, die sonst auf germanischem boden vom neunordischen abgesehen unerhört ist. Dagegen hat die erweichung der spirans nichts auffallendes, und wir werden im weiteren verlaufe unserer untersuchung gelegenheit haben reichliche belege dafur beizubringen. Der hauptgrund aber dafur, dass die indogermanische tenuis bereits zur spirans geworden war, als die erweichung eintrat, ist der, dass dieselbe sich im gotischen als eine ganz junge lautveränderung erweist, die noch im werden begriffen ist, und der deshalb nur diejenige lautstufe zu grunde liegen kann, auf welcher das gotische zur zeit der entstehung der uns uberlieferten denkmäler stand. Der beweis dafur liegt in folgendem. Schon der ganz lebendige wechsel zwischen aus und inlaut weist darauf hin, dass dazwischen keine andern unterschiede bestanden als das vorhandensein oder fehlen des stimmtons. Auch in den hierher gehörigen wörtern wird nicht selten im auslaut die media geschrieben, was, da an dieser stelle wol an eine erweichung nicht zu denken ist, nur aus der spirantischen natur derselben zu erklären ist. Hier findet sich auch $h$ im auslaut neben $g$ im inlaut in aih-aigum und $f$ neben $b$ nach $r$ in parf-baurbum. Ferner findet sich ein schwanken in ganz nahe verwanten wörtern, wie juggs--juhiza, huggrjan - huhrus, vigans - veihan, filhan - fulgins und filigri, fagrs - gafahrjan, faginon - faheps, tigus - taihun, alds - alpeis, stap, stadis - im compositum lukarnarstada, naupjan-naudibandi und anderen (vgl. Holtzm. altd. gr. 22. 29); ja in einem und demselben 
worte: aihan - aigan, gupa-guda; und in einem und denselben suffix: brothar - fadar, missadedins - gabaurpai, gabaurjobus auhjodus, diupipa-junda, fijajva-jivadv, unbarnahs-vuljags (vgl. Lottner a. a. o. s. 194. ff.). Am deutlichsten schen wir noch die entstehung des $b$ aus $f$ in den schon oben angefuihrten fremdwörtern asabis und ioseba und in den praepositionen af und uf, die bei antritt des suffixes uh sofort zu $a b u h$, ubuh werden. Die weitere betrachtung wird zeigen, dass die erweichung in den ubrigen germanischen sprachen, von denen wir erst aus späterer zeit denkmale besitzen, noch weitere fortschritte gemacht hat. Auch im gotischen selbst können wir an den von lateinischen schriftstellern uberlieferten eigennamen den immer weiter greifenden übergang von inlautendem th $\mathrm{zu} d$ beobachten. Ich denke, alles diess tut zur genüge dar, dass sich im ganzen inlautendes $g, d, b$ ron $h, b, f$ nur durch den stimmton unterschieden haben. Schwierigkeiten macht allerdings das $h$, für dessen aussprache als blosser hauch, wie schon bemerkt, entscheidende gründe vorliegen. Doch ist dieselbe hauptsächlich nur für den anlaut gesichert. Im inlaut muss es wenigstens vor consonanten, wenn es überhaupt hörbar werden sollte, voch reibelaut gewesen sein und es bleibt die möglichkeit, dass es auch zwischen vokalen noch stärker gesprochen wurde. Für unseren zweck genugt es, dass wenigstens die aussprache des $h$ als reibelaut die allernächste vorstufe war und dass gar nicht daran zu denken ist, dass es aspirata oder affricata gewesen sei. Ebenso wird wol von niemand bezweifelt, dass $f$ reine spirans war. Nur $b$ ficht man immer an. Aber wir haben widerum gesehen, dass es sich in jeder beziehung dem $f$ vollkommen analog verhält. Scherer nimmt auch an, dass $g$, $d, b$ aus spiranten orweicht soien, meint aber, dass sic damn durch denselben akt wie die indogermanischen medienaffricaten, die er statt der aspiraten annimmt, zu medien verschoben seien, und setzt diesen akt an den schluss der germanischen lautversehiebung, so dass die erweichung zwischen dieselbe fallen würde. Das geht aber ében deshalb nicht an, weil wir die erweichung in den ältesten auf uns gekommenen denkmälern noch im werden begriffen sehen und sie nicht eine geraume zeit zurückschieben können. Auch haben wir kein recht tönende spirans und medienaffricata ohne weiteres zu identificie- 
ren und durch denselben process aus ihnen die media entstehen zu lassen. Vielmehr fällt die erweichung wirklich nach allen den veränderungen, die wir unter dem namen ngermanische verschiebung " zusammenfassen können, nur dass das resultat derselben, sei es aus den medienaspiraten oder aus medienaffricaten, im inlaut nicht medien, sondern tönende spiranten sind, und die aus der erweichung entstandenen laute waren wirklich noch zu Ulfilas zeit tönende spiranten, was Scherer läugnet, und fielen deshalb mit den schon fruher vorhandenen zusammen. Vor der verschiebung derselben zu medien trat die erweichung allerdings ein. Aber diese erfolgte, wie wir sehen werden, erst viel später und durchgängig nur im hochdeutschen. Soweit können wir schon durch die betrachtung des gotischen allein kommen; die der ubbrigen dialekte wird uns diess resultat bestätigen.

Ich führe noch ein paar weniger bedeutende tatsachen an, die für die spirantische natur von $g, d, b$ sprechen können. In den eigennamen aus späterer zeit wird agi in ai, igi in $i$ zusammengezogen (Dietrich s. 73.74). Die zwischenstufen mulssen aji und $i j i$ sein, und darin kann das $j$ am leichtesten aus dem gutturalen spiranten entstanden sein. Möglich bleibt es allerdingis, dass der einfluss des $i$ auch den gutturalen verschlusslaut zum palatalen spiranten umgewandelt hätte, jedenfalls aber ist es minder wahrscheinlich. - In einem falle ist $d$ in $z$ uberyegangen, nämlich in izvar. Das beweist an. yðar, welches, wie schon das $y$ zeigt, aus yðvar $=i \succsim v a r$ hervorgegangen ist. Nicht $z$, sondern $\partial$ ist das ältere. Die vergleichung des ahd., as., ags. ergibt als grundform ivar; $\partial$ ist eingeschoben wie in batimr und wic sonst $d d$ oder gg. Nach unserer auffassung hätten wir dann in izvar aus idvar nur den thbergang von dentalem $s$ in alveolares, resp. dorsales $s$. Fir $b$ endlich ist noch anzuftuhren, dass es in fragibtim luc. 1,27 die stelle von $f$ vertritt und in dem suffix $\left.u b n i^{*}\right)$ mit $f$ wechselt (Holtzm. altd. gr. 33).

*) Dasselbe ist iiberhaupt rätselhaft. Da es im indogermanischen keine suffixe mit lippenverschlusslauten gibt, so muss $b$ oder $f$ wol aus einem andern laute entstanden sein. Nun findet sich sonderbarer weise im altn. nafn und safna neben den sicher älteren namn und samna, während sonst umgekehrt $m n$ neben älterem $f n$ steht. Ist diess nicht für etwas rein orthographisches zu nehmen, so könnte man vermuten, 
Wir wenden uns nun zu den tabrigen dialekten. Es wird sich empfehlen die betrachtung der labialen voranzustellen, da bei diesen das verhältnis am klarsten zu tage liegt. Im altnordischen entspricht gotischem $f$ uberall $f(m f$ ist zu $m m$ geworden in $f \mathrm{~mm}$ ), gotischem $b$ anlautend $b$, inlautend $f$; nur nach $m$ steht auch $b$ und ausserdem findet sich $b b$ im inlaut in wörtern, die im gotischen keine entsprechungen haben. Man findet in sprachwissenschaftlichen werken gewöhnlich angegeben, altn. $f$ sei nur lautgesetzlicher vertreter für $b$ und hält sich mit dieser redensart aller weiteren untersuchung des verhältnisses für uberhoben. Nun ist aber klar, dass der wechsel von $b$ und $f$ der für das gotische nachgewiesenen verschiedenheit in der aussprache des $b$ entspricht. Der unterschied ist hier nur noch klarer und auch durch graphische verschiedenheit bezeichnet. Dass $f$ nur eine spirans ausdrticken kann, ist selbstverständlich und aus der sichern unterscheidung von $b$ und $f$ im anlaut geht anderseits hervor, dass ersteres immer den verschlusslaut bezeichnet. Eine abweichung vom gotischen besteht darin, "dass auch nach $l$ und $r$, nach denen sich im gotischen das $b$ analog dem nach $m$ zu verhalten scheint, $f$ herscht, wofur nur vereinzelt $b$ eintritt (vgl. Holtzm. altd. gr. 117). Es fragt sich nun: haben wir $f$ in diesen fällen als tonlos zu fassen, worauf der buchstabe zunächst hinweist. An und für sich ist es im höchsten grade unwahrscheinlich, dass ein verlust des tones im inlaut zwischen vokalen stattgefunden haben sollte. Anderseits begreift sich die anwendung des eigentlich für den tonlosen laut bestimmten zeichens auch für den tönenden sehr wol aus dem mangel eines eigenen verfügbaren zeichens für den letzteren. Denn $v(u)$ war fur den dem got. $v$, ahd. $w$ entsprechenden laut in anspruch genommen, der sicher im älteren altnordisch noch die von Brucke $\$ 70$ beschriebene halbvokalische natur hatte.*) Um so weniger konnte dasselbe fur die rein

dass auch im got. $m n$ zu $f n$ oder $b n$, d. h. zu labialer spirans $+n$ geworden wäre, so dass dann z. b. vitubni abgesehen vom genus ganz gleich dem lat. calumnia gebildet wäre.

*) Dass diese aussprache des $v$, sowie die entsprechende des $j$ die ursprüngliche indogermanische gewesen ist, erhellt aus einer reihe von erscheinungen in den verschiedenen sprachen und wird wol von niemand bezweifelt. Dass diese aussprache anch noch im germanischen lange fort- 
consonantische spirans gebraucht werden, wenn dieselbe etwa, woruber sich etwas sicheres kaum wird ermitteln lassen, bereits wie in den neueren nordischen sprachen labiodental geworden war. Für die nichtunterscheidung von tönender und tonloser

gedauert hat, dafiir sprechen namentlich folgende gründe: 1) Im englischen besteht sie noch heute im anlaut, während im in- und auslaut das $w$ vollständig vokalisiert oder ausgefallen ist. 2) Im gotischen ist noch das indogermanische gesetz des weohsels von $u$ und $v$, je nachdem consonant oder vokal folgt, vollständig lebendig, was fiir die grosse leiohtigkeit des iiberganges spricht. 3) In gotischen eigennamen wird von lateinischen schriftstellern das anlautende $v$ durch $u u, v v, u v . u b$ widergegeben, eine schreibung, wie sie fiir den aus mit einander verschmolzenem vokal und consonant bestehenden laut sehr geeignet ist; vgl. dariber Dietrich auspr. 78. 79. Wenn solche schreibungen nicht auch im inlaute vorkommen, so ist dadurch noch nicht bewiesen, dass hier rein consonantische aussprache galt wie Dietrich behauptet; dagegen spricht das unter 2. angefiihrte gesetz und der umstand, dass in anderen dialekten gerade im anlaut, nicht im inlaut der vokalklang verloren geht; 4) Gotisches $v$ erscheint in den eigennamen vor folgendem $u$ oder $i$ mit diesen zu $u$ verschmolzeu vgl. Dietr. 79. 80. 5) In allen germanischen sprachen finden sich verschmelzungen des $(w)$ nach $k$ oder $s$ mit dem folgenden vokal zu $u$ oder $o$. 6) Die im ahd., as. und zum teil im ags. gebräuohliche schreibung $u u$, woraus sich dann $w$ entwickelt, ist ebenso durch die aussprache berechtigt, wie in den gotischen eigennamen; 7 ) Wenn im ahd., as., afr., ags. ein kurzer vokal vor $w$ zum diphthongen wird so folgt das fast notwendig aus dessen vokalischer natur; das $u$ des diphthonges ist der vokalische bestandteil des $w$, der auch schon im gotischen vorhanden war und nur allmählich etwas stärker hervorgetreten zu sein und sich enger mit dem vorhergehenden vokal verbunden zu haben scheint. 8) Auslautend wird $w$ im ahd. und as. zu $u$ oder $o$, d. h. es bleibt nur das vokalische element, das consonantische ist zu schwach sich zu halten. 9) Die schwäche des consonantischen elements zeigt sich dann weiter darin, dass es spüter im inlaut zwischen vokalen ganz ausfällt, während das vokalische nach kurzem vokal und auch nach langem $a$ in der mit diesem eingegangenen diphthongischen verbindung erhalten bleibt. Der schwund des consonanten ist im mhd. wahrscheinlich in der aussprache frllher eingetreten als in der schrift. Denn im 15. jahrh. wird ganz gewöhnlich so gut wie frawe auch haws, awsz u. dergl. geschrieben. 8peciell für die aussprache des altn. ist von bedeutung: 1. dass es überall vor dunkeln vokalen ausfällt; 2. dass es im auslaut und vor dem nominativs $r$ nach langem $a$ und $\hat{\imath}$ zum vokal wird, sonst aber abfällt; 3. dass Thorodd in seinem traktat über die isländischen buchstaben noch gar keinen consonanten $u$ oder $v$ kennt. Das $u$ ist $\mathrm{thm}$ wie das $i$ nur in soweit consonant, als es thm auch sonst ein mit einem ondern zum diphthongen verbundener vokal ist. 
spirans werden wir alsbald weitere belege finden. $\mathrm{Es}$ ist ja auch nichts anderes, wenn wir im nhd. kein besonderes zeichen für das weiche $s$ haben. Für tönende aussprache des dem gotischen $b$ entsprechenden $f$ spricht erstens, dass es vokalisiert wird in haukr, Giuki; ferner der wechsel von $f$ und $m$, wobei bald das eine, bald das andere ursprunglich ist. Derselbe ist gewöhnlich vor $n$, kommt aber auch in einigen andern fällen vor wie in hifinn, helfingr vgl. Holtzm. 118. Da $m$ tönend ist, muss auch der mit ihm wechselnde consonant tönend sein. Ein beweis für tönende aussprache des $f$ ist ferner das eintreten desselben furr $v$ besonders nach langem vokale. Darin hat man nicht einen verlust des stimmtons zu sehen, wogegen schon die moderne aussprache spricht, sondern die ersten spuren der verwandlung des halbvokals in einen reinconsonantischen, vielleicht labiodentalen laut. Weiter führt uns die betrachtung der neunordischen sprachen. Im isländischen bleibt in der schrift uberall $f$, wird aber im in- und auslaut ausser vor tonlosen consonanten, gleichviel ob es gotischem $f$ oder $b$ entspricht, tönend wie deutsches $n$ gesprochen, nur vor $l, n$, $\partial$ wie $b$. Im schwedischen tritt inlautend $f v$, auslautend $f$ ein, im dänischen (auch im færœischen) in - und auslautend $v$. Die aussprache ist in beiden in - und auslautend $=$ deutschem $n$. Diese ibereinstimmung der verschiedenen zweige des nordischen ist ein hinlänglicher grund die durchgängig tönende aussprache des $f$ im in- und auslaute für alt zu halten, wie auch Wimmer tut. Dass die abweichende aussprache des isländischen vor $l, n, \delta$ erst eine jüngere veränderung ist, beweist schon die schrift und anderseits die nichtubereinstimmung des schwedischen und dänischen. Ziemlich alt mag sie aber doch sein, wie die von Holtzmann auf s. 117 angefuhrte schreibung Fabnir beweist. Wir haben also folgendes resultat gewonnen: urgermanisches $f$ ist im nordischen in- und auslautend ausser vor tonlosen consonanten tönend geworden und dadurch mit der schon fruher bestehenden tönenden spirans zusammengefallen. Nun erklärt sich um so mehr die anwendung des zeichens $f$ fur beide.

Analog ist das verhältnis im angelsächsischen. Hier vertritt ebenso $f$ got. $f$ uberall und got. $b$ im in - und auslaute, ausgenommen nach $m$ und in der gemination, wo wie im anlaute $b$ steht. Auch hier kann die tönende aussprache des $f$ 
für den inlaut nicht zweifelhaft sein. Erstlich wechselt es an dieser stelle mitunter mit $v$, welches besonders in den Northumbrischen evangelien häufig ist; $v$ bezeichnet aber im ags. und ergl. stets den weichen laut $==$ lat. und franz. $v$. Es findet sich ferner ein paar mal $b$ (Holtzm. 217), gewis ein zeugnis für tönende aussprache. Kaum wahrscheinlich aber ist es, dass damit ein explosivlaut hat bezeichnet werden sollen; jedenfalls würde dieser nicht etwa altertümlich sein, da sich auch einmal färbu statt farewu findet. Weiter ist beweisend der ubergang von $f$ vor $n$ zul $m$, wie im altu. Dass auch das got. $f$ entsprechende $f$ tönend war, geht daraus hervor, dass es bei eintretender gemination $\mathrm{zu} b b$ wird in hebban $=$ got. hafjan. Das entscheidendste aber ist wider las lautverhältnis in den jüngern sprachperioden. Im neuangelsächsischen, alt- und mittelenglischen wird allmählich das $f$ im inlaut vom $v$ gänzlich verdrängt, was gewis mit der massenhaften einfihhrung romanischer wörter zusammenhängt, wodurch $v$ ein geläufiger buchstabe wurde; in neuengl. steht es therall, wo ursplinglich $f$ inlautend war, nit dem laute der labiodentalen spirans. Dagegen wo $f$ im ags. auslautend war, da steht abweichend vom nordischen im neuengl. tonloses $f$, scheinbar nun auch im inlaut in wörtern wie life, wife, in denen aber das stumme $e$ nur angehängt ist zum zeichen, dass $i$ diphthongisch $z u$ sprechen ist. Diese abweichung wird, da nichts dagegen spricht, ebenfalls alt sein. Für das angelsächsische ist daher die regel so zu fassen: germanisches $f$ wird im inlaut erweicht und fällt dadurch mit der tönenden spirans $=$ got. $b$ zusammen, während umgekehrt im auslaut letztere den stimmton verliert und so gleichfalls mit dem alten $f$ zusammenfällt.

Im altsächsischen sind gleichfalls got. $b$ und $r$ in- und auslautend zusammengefallen. Im Heliand steht im auslaut für heide in der regel $f$, daneben $\hbar$ und $b$, inlautend $f$ nattirlich immer vor tonlosen consonanten, in der regel auch vor $n$ und I, sonst aber ist das eigentlich regelmässige $b$, daneben findet sich $b$, namentlich im Monacensis, und $v(u)$, sehr selten $f$, vereinzelt $u$ in hiouuandi $=$ ahd. hiufanti 5516 Cott.; in der gemination steht ausnahmslos $b b$, welches dann im auslaut vereinfacht wird. Verschieden entwickelt sind nur gerade wie im ags. die gotischen lautverbindungen $m b$ und $m f$. Ersteres ist 
auch im alts. consequent $m b$, in letzterem schwindet der nasal und das $f$ wird dann auch im inlaut $z u b$. In den ubrigen altsächsischen denkmälern findet sich kein $\mathrm{t}$, sondern statt dessen durchgängig $v$, ebenso im altfriesischen und im altniederfränkischen, auch im altmittelfränkischen, welches durch das 'Trierer capitulare vertreten wird,*) während die aitesten denkmäler des sud - und ostfränkischen wie das oberdeutsche $b$ und $f$ streng scheiden. Es ist nicht denkbar, dass die verschiedenen zeichen im Hel. wirklich verschiedene laute bedeuten; denn sie wechseln ganz beliebig mit einander. Wir können vielmehr darin nur verschiedene versuche \%ur bezeichnung desselben lautes sehen und mussen fur den inlaut sowol als für den auslaut je eine gleichmässige aussprache annehmen, die sich auch mit ziemlicher sicherheit wird feststellen lassen. Dass $f$ und $v$ nur eine spirans bezeichnen können, ist klar, und zwar wird man von letzterem, solange nichts anderes dagegen spricht, annehmen, dass die tönende damit widergegeben werden soll, wiewol es allerdings später auch für die tonlose verwendet wird. Anderseits weisen ebenso sicher $b$ und $b$ auf einen tönenden laut hin. $b$ ist jedenfalls vollkommen analog dem $\partial \mathrm{zu}$ beurteilen; die bestimmung des lautwertes des einen muss massgebend für den des andern sein. Die spätere betrachtung des $\partial$ wird unsere auffassung des $b$ als tönende spirans nur stutzen. Die erfindung dieses zeichens wurde wol dadurch veranlasst, dass $v$ im lateinischen labiodentale spirans ist und deshalb das zeichen für den deutschen ursprunglich sicher rein labialen laut nicht gentigend erschien. Wenn daftir auch $b$ geschrieben wird, so könnte man darin vielleicht mit Holtzmann hochdeutschen einfluss sehen, wahrscheinlich aber ist es nur nachlässige schreibung wie $d$ fur $\delta$. In keinem falle aber haben wir darin einen älteren lautstand zu erkennen; denn es steht ebenso gut fur got. $f$, als fur $b$. Dass im auslaut $f$ bei weitem uberwiegt, zeigt uns, dass hier verhärtung der weichen spirans eingetreten ist. Wenn daneben $b$ und $b$ sich findet, so haben wir diess wol

*) Auch in dem in das nördliche Thüringen oder Hessen gehörigen gedichte de Heinrico findet sich $v$ und $f=$ got. $b$; fur die entsprechung des got. $f$ findet sich kein beispiel ausser ovar, in welchem worte auch abd. orweichung eingetreten ist. 
nicht anders aufzufassen, als wenn im gotischen $b$ und $d$ im auslaut bleibt. Die verhärtung verstand sich so von selbst, dass sie durch die schrift nicht angedeutet zu werden brauchte, gerade wie sie im nhd. niemals bezeichnet wird. Demnach hätten wir im altniederdeutschen genau dasselbe verhältnis wie im ags.

Auch in der folgenden zeit bleibt diess unverruckt dasselbe. Im mittelniedeideutschen und niederländischen wird im inlaut ausser nach $m$ und in der gemination, wo $b$, und vor harten consonanten wo $f$ steht, consequent $v$, im auslaut $f$ geschrieben sowol für got. $f$ als $b$, und beide arten von $f$ und $v$ reimen unbedenklich auf einander. In der heutigen aussprache klingen sie wie neuhochdeutsches $w$ und $f$, als tönender und tonloser labiodentaler reibelaut. Wenigstens ist mir nicht bekannt, dass labiolabialer laut irgendwo vorkäme. Mit der endsylbe en verschmilzt dies $n$ (ich weiss nicht, ob in ganz Niederdeutschland) zu einem sylbenbildenden $m$, \%. b. ge-m fur gewen. Da dieses $m$ auch von den hochdeutsch redenden niederdeutschen gesprochen wird und diese sich dessen nicht bewust sind, vielmehr nach der schrift ben zu sprechen glauben, so entsteht der irrtum, als spräche man in diesem falle auch plattdeutsch ein $b$, was man sogar in mundartlichen grammatiken angegeben findet, z. b. in Nergers grammatik des Meklenburger dialekts S 189 . 191. Ueber das holländische $v$ werden die allerverschiedensten angaben gemacht, vgl. darubber Ruinpelt, system der sprachlaute s. 60. 61 und Scherer, zeitschr. f. d. östr. gymn. XII, 635. Es wird doch wol zwischen an- und inlautendem zu unterscheiden sein, und vielleicht beruhen die widerspruche in der angabe der aussprache auf der ausserachtlassung dieses unterschiedes. Das mittelfrünkische schliesst sich in diesem punkte ganz dem niederdeutschen an. Aber noch viel weiter sudlich geht sowol der gebrauch der spirans für got. $b$, als die erweichung des got. $f$ im inlaute. Die dinge liegen hier nicht so einfach wie im niederdeutschen, und verschiedene schwierigkeiten sind in erwägung zu bringen. Es wird sich empfehlen vom lautstande der heutigen sprache auszugehen, wenn man zu einem urteil uber die verhältnisse in der älteren zeit gelangen will. Leider fehlt es so sehr an einer zuverlässigen beschreibung der laute der einzelnen mundarten, dass einstweilen manches unsicher bleiben 
muss und ganz sichere grenzbestimmungen sich nicht angeben lassen. Soviel ich dartiber ermitteln konnte, wird tönende spirans für got. $b$ im inlaut gesprochen in Südfranken, Hessen, Thüringen, Obersachsen, auch noch weit nach Oberdeutschland hinein im Elsass in Schwaben und Baiern. In Schlesien findet sich nach Weinhold (laut- und wortbildung und die formen der schlesischen mundart p. 72.) $w$ nur in der gegend von Mittelwalde, anderswo aber uberall $b$. Dagegen behauptet Rückert, (Entwurf einer darstellung der schlesischen mundart im mittelalter, zeitschr. des vereins f. gesch. I. altert. Schlesiens IX. p. 36), dass allgemein $w$ gesprochen wïrde, ein recht schlagender beweis dafür, wie unzuverlässig alle angaben über aussprache sind. Nicht so weit erstreckt sich die erweichung des $f$. Sie ist durchgeführt in Südfranken, Hessen, Schlesien, nicht in Obersachsen abgesehen vom nördlichen teile, ich glaube auch nicht in Thüringen, so dass in Süidfranken und Hessen und in Schlesien, soweit $w$ für $b$ gesprochen wird, got. $b$ und $f$ im inlaut wie im niederdeutschen in einen laut zusammengefallen sind. Es handelt sich nun darum, wie weit dieser lautstand alt ist. Ich habe schon bemerkt, dass im altstidfränkischen $z$. b. bei Otfrid $b$ und $f$ noch scharf geschieden sind. Die erweichung des letzteren muss hier erst später eingetreten sein, ist es aber sicher im XII. jahrh. Noch später scheint sie in Schlesien erfolgt zu sein. Denn in den dahin gehörigen denkmälern wird das ganze mittelalter hindurch fast consequent im inlaut $f$ geschrieben (vgl. Rtickert a. a. 0. 34), gerade wie in den obersächsischen.

Was das $w$ betriffit, so ist an und fir sich naturlich tuberall die möglichkeit, dass es erst aus dem verschlusslaut entstanden ist. Lnd dafür scheint zu sprechen, dass in diesen gegenden uberwiegend $b$, seltener $v$ geschrieben wird, und dass namentlich Otfried nur $b$ kennt. Anderseits aber steht $b$ da, wo erweichung des $f$ eingetreten ist, ebenso gut auch für dieses, namentlich in den siidfränkischen urkunden, aber auch in handschriften von gedichten, z. b. im Alexander, in Hartmanns Credo, bei Herbort von Fritzlar, in der Elisabeth (vgl. Reissenberger tiber Hartmanns rede vom glauben 29), auch bei Nicolaus von Jeroschin (Pfeiff. LXIV.). Beicle arten von $b$ reimen unbedenklich auf einander. Man würde also zu der unwahr- 
scheinlichen ammahme getricben, dass die aus $f$ erweichte spirans erst zum rerschlusslaut und damn wider zur spirans geworden wäre. Weiter tritt las $b$ auch für $w$ ein rgl. Pfeiffer zu Jeroschin LXIV, Rüuckerí a. a. o. 31, während es heute wenigstens nur in einzelnen landschaften gesprochen wird. Bei Jeroschin und in Hartmanns Credo reimen $b$ und $w$ auf einander. Da nun anderseits auch $v$ für $b$ nicht selten ist und auch $w$ dafür geschrieben wirl, so scheint es mir das wahrscheinlichste, dass inlautendes $b$ im mitteldeutschen die spirans bedeutet. Diese lautbezeichnung begreift sich, wenn man bedenkt, dass rein labiale spirans gesprochen wurde, welehe dem verschlusslaut $b$ eben so nahe steht, als dem labiodentalen lateinischen $v$, welches noch dazu, wie wir später sehen werden, für die tonlose spirans verwendet zu werden anfing. Und $w$ konnte dafür erst eintreten, nachdem es sein vokalisches element verloren hatte. Noch heute wird in Mitteldeutschland, wenigstens in Obersachsen, Thüringen, Hessen, Frankfurt nur labiolabiales $w$ sowol für. altes $w$, als für got. $f$ und $b$ gesprochen, im anlaut wol meistens mit fliusterstimme.*) In Süddeutschland muss das $w$ erst vor kurzem labiodental geworden sein, da, worauf Scherer aufmerksam gemacht hat; noch im ausgange des vorigen jahrhunderts der physiologe Kempelen in Wien nur labiolabiales $w$ kennt. Dagegen geht in Norddeutschland, soviel ich weiss, das labiodentale $n$ durch. Ist dieser unterschied alt, so liegt es nahe zu vermuten, dass die in Mitteldeutschland tiberwiegende schreibung mit $b$ gegenúber dem niederdeutschen und mittelfränkischen $v$ eben daraif beruht. Allerdings ist ein bedenken gegen das hinautrücken der labiodentalen aussprache in ein so hohes alter, nümlich ller ihergang von ven in $m$, wolcher doch fast die labiolabiale aussprache des $v$ vorauszusetzen scheint. Wer nun diese argumentation nicht für überzeugend hält und meint, das $b$ überall, wo es geschrieben wird, einen verschlusslaut bezeichne, der muss zugeben, lass $b$, wo es got. $f$ oder $v$

*) Ich behalte diesen ausdruck bei, weil er jetzt ziemlich eingeführt ist, wiewol die natur dieser laute vielleicht noch anders zu bestimmen sein wird. Auch in Niederdeutschland wird das $w$ zum teil mit fluisterstimme gesprochen, gleichviel welchen ursprunges es sei, wahrscheinlich in denselben gegenden, in denen man anch gefliisterte medien spricht. 
entspricht, aus einem reibelaut entstanden sein $\mathrm{muss}$, und es deshalb auch da, wo es got. $b$ entspricht, sein kann. Wird in Schlesien wirklich $b$ gesprochen, so wird diess eben so gut erst aus der spirans geworden sein, wie es sehr häufig aus altem $w$ entsteht, z. b. in ebich, löbe vgl. Weinhold, schles. mundart 75. Auch andere mitteldeutsche dialekte kennen die verwandlung des $w$ in $b$, auch im anlaute. Jedenfalls beweist also das vorkommen von $b$ im inlaut $=$ got. $b$ nichts gegen die urspringlichkeit des reibelautes.

Im auslaut wird im mitteldeutschen, abgesehen vom mittelfränkischen, wo $f$ die regel ist, entweder $b=$ got. $b$ beibehalten oder $p$ geschrieben. Heutzutage wird $p$ gesprochen. Nur in Schlesien wird, wie Weinhold und Ruckert behaupten, nach langem vokal die media (auch $d$ und g) gesprochen. Nach den unbestimmten angaben von Rückert, a. a. o. s. 334 scheint es aber, als ob das vielmehr die unaspirierte tenuis ist. Sicher ist also von alters her verschlusslaut gesprochen worden. Diess scheint in widerspruch zu stehen mit unserer auffassung des inlautenden $b$, da wir nach dem allgemeinen gesetze entsprechung des in- und auslautes erwarten müsten. Da aber in den neuern mundarten keine genaue entsprechung stattfindet, so haben wir ein recht, diess auch für die ältere zeit anzunehmen. Und dieses misverhältnis lässt sich sehr wol begreifen. Der labiolabialen tönenden spirans im inlaute sollte die labiolabiale tonlose im auslaute entsprechen. Dieser schwierige laut ging im niederdeutschen in den labiodentalen laut uber, indem vielleicht der inlaut gleichzeitig dieselbe wandlung durchmachte, im mitteldeutschen in den ihm eben so nahe stehenden verschlusslaut. Diese auffassung wird dadurch bestätigt, dass auch statt des got. $f$ in Sudfrankeu und Hessen sehr gewöhnlich $p$ resp. $b$ geschrieben wird. Es reimen in Hartmanns Credo brief: lieb 1980, bei Herbort vrloub: hob 2375, hob: lob 3033 u. a., während anderseits auch brief: lief, so dass man wol annehmen muss, dass er noch spirans sprach. Noch heute wird in Hessen briep, hop gesprochen. Das berechtigt uns das $p$ auch da, wo es got. auslautendem $f$ fur inlautendes $b$ entspricht, als aus der spirans entstanden anzunehmen.

In Oberdeutschland und einem teile von Mitteldeutschland tritt also, wie schon bemerkt, die erweichung des $f$ im allge- 
meinen nicht ein, aber doch in einer bestimmten anzahl von wörtern. Diese haben in den ältesten ahd. denkmälern $v$, und zwar auch schon in solchen, welche im anlaut und auch inlautend in anderen wörtern niemals $v$ haben, so dass hier $f$, $v, b$ deutlich geschieden sind. Dieses $v$ geht dann allmählich in $b$ und sogar im strengalthochdeutschen in $p$ ther. Solche wörter sind z. b. avar avur, später abur, heven - heben, inseven - entseben, hwerfan - werben, eiver (amarus) N. - eibar eipar, diuva (furtum) - diuba, zouver - zouber. Hie und da findet sich in ältester zeit auch in diesen wörtern noch $f$, sogar geminiert in heffan K. 0 . Der ubergang in $p$ oder $b$ ist bereits vollzogen, als unsere uberlieferung beginnt, in ubar = got. ufar, wahrscheinlich weil in diesem die eiweichung fruher eingetreten war, in den ubrigen wörtern beginnt er auch schon wenigstens in der mitte des neunten jahrhunderts, tritt aber nicht uberall gleichmässig ein, sondern $v$ erhält sich daneben auch im mhd. Auch scheinen dialektische abweichungen vorzukommen in bezug auf die wörter, in denen die erweichung eintritt.

Eine grosse verwirrung entsteht nun dadurch, dass bereits im neunten jahrh. auch in allen ubrigen wörtern $v$ für got. $f$ im inlaut sich einzudrängen beginnt, und sich allmăhlich ausser vor $t$ und $s$ so festsetzt, dass daneben $f$ nur noch selten orscheint.*) Es ist eine ziemlich allgemeine annahme, die auch Scherer teilt, dass auch dieses $v$ uberall die tônende spirans bezeichnen solle. Indessen wie erklärt man sich dann das nhd. $f$ ? Ist es denkbar, wenn im mhd. das $v$ in hove, brieve, tavel etc. allgemein tönend gesprochen wäre, dass dann im nhd. die ursprtungliche tonlosigkeit wider; hergestellt wäre?**) Und was fur oinen zwingenden grund hat man dem $v$ hier den stimmton zuzuschreiben? Es tritt ja ron derselben zeit an

*) Doch in handschriften bedeutend häufiger als in unsern kritischen ausgaben mittelhochdeutscher texte.

*) Das nhd. $f$ in diesen wörtern ist keineswegs bloss graphisch zur herstellung der tibereinstimmung mit dem auslaut eingetreten, wie Rumpelt (Das natiurliche system der sprachlaute 59) durch seinen schlesischen dialekt verführt meint, und die tonlose aussprache ist nicht bloss dialektisch und individuell, sondern allgemein als mustergiiltig angenommen. 
auch im anlaut für $f$ ein und wird bis auf den heutigen tag geschrieben. Der unterschied ist nur, dass es im inlaut noch etwas mehr als im anlaut vor dem $f$ uberwiegt. Man muste also behaupten, dass auch im anlaut das $f$ erst erweicht und dann wider verhärtet wäre. Gibt man die tonlose aussprache des $v$ aber für den anlaut zu, so kann man es auch für den inlaut. Die früher angefülırten wörter sondern sich eben dadurch von den übrigen ab, dass in ihnen $v$ schon in denkmälern erscheint, die niemals $v$ für $f$ im anlaute haben, und dass es mit $b$ und $p$ wechselt und dass das erstere in der neuhochdeutschen schriftsprache zur herschaft gelangt ist. Vereinzelt mag die erweichung weiter gegangen sein und ist es in neuern oberdeutschen mundart $n$ entschieden. Aber allgemein ist sie niemals geworden. Man darf sich nicht darauf berufen, dass die unterscheidung zwischen altem $f$ und dem erst durch dic hochdeutsche lautverschiebung entstandenen darauf beruhen mulsse, dass ersteres tönend geworden, letzteres tonlos geblieben sei. Es sind vielmehr andere unterschiede rorhanden. Das neue $f$ ist geminiert, da es durch assimilation aus $p /$ entstanden ist, ursprünglich auch nach langen vokalen, nach welchen sich die gemination nur nach einem allgemeinen gesetze allmählich vereinfachte.*) Wahrscheinlich aber bestand noch eine andere verschiedenheit, die auch zur unterscheidung der einfachen laute gentigte. Das alte $f$ wurde wol fruher labiodental als das neue eben aus $p f$ entstandene und zum teil wie in scharf, gelf u. a.m. noch vor unsern augen entstehende. Uebrigens findet keineswegs eine ganz reinliche sonderung statt. Es findet sich $v$ für neues $f$ (Weinh. bair. gr. 132.) und ph für altes (ib. 129). In dürfen wird, so viel ich weiss niemals $v$ geschrieben und es reimt durfen: wurfen Martina 144, 79. Es scheint also das alte $f$ in diesem worte mit dem neuen zusanmengefallen zu sein. **) Die anwendung des $v$ fur den tonlosen spiranten er-

*) Vgl. d. beitr. p. $48 \mathrm{ff}$.

**) Ganz analog ist das verhältuis zwischen $s$ und $z$, soweit das letztere einen einfachen spiranten, nicht $t+s$ bezeichnet. Scherer (zur geschichte $10.1 \mathrm{ff}$., zeitschr. f. d. östr. gymn. 1870. 756. f.) hat behauptet, dass $s$ im ahd. und älteren mhd. tönend sei und dass es sich dadurch von dem $z$ unterscheide, welches die entsprechende tonlose spirans bedeute. Diese annahme steht im entschiedensten widerspruch mit der hentigen 
klärt sich daraus, dass es in Deutschland ublich war dasselbe auch im lateinischen so zu sprechen, eine gewohnheit, die zum teil bis auf den heutigen tag fortdauert. Nur aus dieser aussprache begreift es sich, dass in dell aus dem lateinischen (später auch in den aus dem französischen) entlehnten wörtern mit $v$ oder nach romanischer weise spirantisch ausgesprochenem $b$ (z. b. tiufal, evangelio) die tonlose spirans eintritt, $f$ oder $v$ geschrieben, während das deutsche doch gar keine neigung zur verhärtung der inlautenden spiranten, sondern im gegenteil

aussprache. In Oberdeutschland (ich weiss nicht ob allgemein) wird noch heute an jeder stelle des wortes scharfes $s$ gesprochen wie sicher im indogermanischen. In der gemination ist, so viel ich weiss, in keiner germanischen mundart erweichung eingetreten. Ist man hier wunderbarer weise wider zur ursprünglichen härte zurückgekehrt? Dasselbe mliste auch im auslaut geschehen sein, wo ja auch $s$ und $z$ in älterer zeit streng geschieden werden. Hier ist ïberhaupt die erweichung vollends undenkbar. Nirgends in Deutschland werden, glaube ich, und wurden schon von alter zeit her tönende verschluss - oder reibelaute im auslaut gesprochen. Vielnehr geht an dieser stelle immer der stimmton, auch wenn er ursprünglich vorhanden war, verloren. Ein sonstiger grund für die annahme von tönendem $s^{\prime}$ liegt nicht vor. Wir müssen nach einem andern unterschiede von $s$ und $z$ suchen. Gegen Rumpelts annahme, dass die aussprache von $z=$ nhd. $s z$ nicht viel verschieden von $t s$ gewesen sei, spricht die analogie von $f$ und $c h(h h)$, wonach wir auch in der dentalreihe hinter vokalen reine spirans erwarten müssen. Der unterschied von $s$ und $z$ kann daher nur auf der verschiedenheit der artikulationsstelle beruhen, und darüber lässt sich, glaube ich, eine ziemlich wahrscheinliche vermutung aufstellen. Es ist bekannt, dass im judendeutsch anch sonst in manchen mundarten und vielfach in individueller aussprache der doppellaut $z$ zu einem gelispelten $s$ wird. Dasselbe ist nach Brickes System als dentales $s$ zu bezeichnen; doch wird bei bildung desselben die zungenspitze nicht so weit vorgeschoben, als bei der des englischen th, sondern kommt höchstens bis an den rand der obern zahnreihe. Dieser laut, welcher physiologisch dem ts zunächst liegt, und den wir noch heute daraus entstehen sehen, muste fast notwendig die übergangsstufe zu dem heute gesprochenen alveolaren oder dorsalen $s$ sein. Mit dem th des ältesten ahd. brauchte derselbe nicht zusammenzufallen. Denn erstlich konnte die artikulationsstelle noch etwas verschieden sein, ferner war $z$ stets, th nie geminiert, endlich ist $t h$ schon in den ältesten quellen als tönender laut zu fassen und im übergang zum verschlusslaut begriffen. Das zusammenfallen des $s$ und $z$ tritt dann um die mitte des dreizehnten jahrhunderts ein, indem letzteres in die artikulationsstelle des ersteren übertritt.

Beitrige zur geschichte der deutschen sprache. I. 
zur erweichung hatte. Die veranlassung zu dieser aussprache war wol, dass die deutschen ohren und zungen an tönende labiodentale spirans nicht gewöhnt waren. Denn $w$ war rein labial und halbvokalisch und unterschied sich ganz scharf von lateinischem $v$. Das aus $f$ erweichte $v$ war zwar rein consonantisch, aber gewis labiolabial, da es bald in $b$ uberging. Dagegen war $f$ wol schon, wie früher erwähnt, labiodental und konnte als der dem lateinischen $v$ zunächstliegende laut angesehen werden und fur dasselbe eintreten. Möglicherweise ist auch die tradition der irischen mönche, welche ihrer muttersprache gemäss das lateinische $v$ verhärteten, von einfluss gewesen. Wir werden also daran festhalten, dass die erweichung $\operatorname{des} f$ im inlaute, welche im altnordischen, angelsächsischen, niederdeutsehen und einem teile des mitteldeutschen durchgedrungen ist, in Oberdeutschland und einem teile von Mitteldeutschland nur einzelne wörter ergriffen hat, während die anderen davon verschont geblieben sind.

Wir kommen jetzt zu der wichtigen frage: kann der hochdeutsche verschlusslaut im inlaut $b$, wie die herschende ansicht dartber ist, im vergleich $\mathrm{zu}$ der von uns in den übrigen dialekten nachgewiesenen spirans als altertumlich betrachtet werden, oder ist er aus dieser hervorgegangen? Die tibereinstimmung sămmtlicher tubrigen mundarten, von denen noch dazu allgemein anerkannt ist, dass sie sonst in bezug auf die consonanten einen alteren lautstand repräsentieren, spricht auf das stärkste für die letztere annahme. Sie würde ein unumstösslicher beweis sein, wenn man an der theorie vom stammbaume festhält, also annimmt, dass nach der trennung des nord- und stidgermanischen beide sich selbständig entwickelt hätten und nicht teile des einen in gemeinschaft mit dem andern lautveränderungen hätten durchmachen können. Ich bin von der unhaltbarkeit dieser theorie uberzeugt und halte es an und für sich wol für möglich, dass eine zusammenhängende lautbewegung $b$ in den ubrigen dialekton zur spirans wandeln konnte ohne das hochdeutsche zu beruhren. Aber die grössere wahrscheinlichkeit ist nattrlich fur die entgegengesetzte ansicht, so lange nichts anderes dagegen spricht. Entscheidender ist folgendes. Wir haben gesehen, dass im got. ein teil der inlautenden $b$ durch erweichung aus $f$ entstanden ist. Dieselben wörter zeigen nun auch 
im ahd. von anfang an $b$ oder $p$, woraus hervorgeht, dass diese erweichung nicht bloss gotisch, sondern uberhaupt germanisch war. Abweichend vom ahd. sind im got. erweicht nur paurban, ainlibim, ivalib, hvairban (in letzterem ist im ahd. die erweichung später eingetreten), abweichend vom got. im ahd. ubar; ebar und intsweben (sopire) fehlen im got. Diese erweichten laute waren also im ahd. wie im gotischen sicher einmal spiranten. Wir müssen also dieser wegen annehmen, dass vor der zeit, aus der uns die ältesten denkmäler erhalten sind, eine allgemeine verschiebung von tönender labialer spirans in tönenden verschlusslaut, der dann zum teil weiter zu tonlosen verschoben ist, stattgefunden hat. Wir können diese verschiebung wenigstens an einem worte aus dem hochdeutschen selbst erweisen, nämlich in dem volksnamen Suevi, Swâbe, Swâpe. Leider ist die etymologie des wortes dunkel, so dass wir nicht wissen können, ob das $v$ indogermanischem bh oder $p$ entspricht. Es scheint aber fast bedenklich die erweichung des $f$ in eine so frühe zeit hinaufzurlicken. Jedenfalls so viel ist klar: derselbe akt, welcher die aus $f$ erweichte spirans verschob, konnte auch den indogermanischem $b h$ entsprechenden laut verschieben, wenn derselbe wie in den tibrigen dialekten tönende spirans war. Diese lautbeweguug dauert nun noch fort in der periode, deren geschichte wir beobachten können. Nachdem von neuem eine anzahl $f$ erweicht waren, machten auch sie, wie wir sahen, die entwickelung zu tönendem und tonlosem verschlusslant durch. Endlich nachdem das $w$ seine vokalische natur verloren hatte, ward auch diess zum teil zu $b$. In bairischen handschriften wird im 14. und 15. jahrhundert unendlich häufig $b$ für $n$ geschrieben, viel seltener im alemanischen. Ich kann mich aber nicht entschliessen zu glauben, dass damit wirklich der tibergang in den explosivlaut bezeichnet werden soll, da sich in der heutigen mundart nur wenige spuren davon finden. Wahrscheinlicher scheint es mir, dass diese schreibung nur den verlust des vokalischen elementes andeutet, und dass $b$ als rein labiale spirans zu fassen ist wie im mitteldeutschen. Lautlich mit $b$ zusammengefallen muss allerdings diess $w$ im inlaute sein; dass beweisen die von Weinhold bair. gr. 125 angefuhrten reime. Aber ich möchte eher glauben, dass $b$ im inlaut zur spirans geworden war, wie es in den neuern bairischen mund- 
arten vielfach der fall ist, oder vielleicht, dass auch hier die spirans von alters her bewahrt ist, wortber ich noch keine entschiedene meinung zu äussern wage. Diese erscheinung möchte ich also nicht zur stlitze meiner ansicht gebrauchen. Sicher aber wird $w$ nach $r$ und $l$, nach denen es ebenso wie $j$ schon fruh seine vokalische natur verloren zu haben scheint, zu $b$, und diess hat sich auch in der nhd. schriftsprache festgesetzt. Vereinzelt kommt $b$ für $w$ auch sonst in neuern mundarten vor (vgl. alem. gr. 155. bair. gr. 124. 125), allgemein auch im anlaute ist es bei den Vicentiner und Veroneser Deutschen. Ich denke, das wird gentugen, um jedes bedenken, das etwa jemand wegen des tubergangs der spirans in den verschlusslaut haben könnte, zu beseitigen. Vielleicht lässt sich auch die in den ubrigen dialekten bestehende verschiedenheit zwischen an- und inlaut noch im hochdeutschen nachweisen. Im ahd. wird im inlaut viel seltener $p$ geschrieben als im anlaut. Es gibt quellen, die es im anlaut häufig und im inlaut gar nicht haben. Im mhd. wird es ausser vor $s$ und $t$ im inlaut fast gar nicht mehr geschrieben. Es ist indessen schwierig daraus einen ganz bestimmten schluss zu ziehen, da im allgemeinen zwischen anund inlaut in ältester zeit nur ein gradueller unterschied besteht, und da das schwanken der schreibung verschiedener deutung unterliegt. Scherer glaubt, dass dadurch die geflusterte media angedeutet wurde. Ich habe (Gab es eine mhd. schriftsprache $24 \mathrm{ff}$.) auszufuhren versucht, dass auch $b$ im anlaut die bedeutung einer tenuis hat. Ihr dieselbe danach auch durchweg fur den inlaut zuzuweisen wurde an und für sich nicht vollkommen ungerechtfertigt erscheinen. Doch scheinen die neuern mundarten dagegen $\mathrm{zu}$ sprechen, deren laute wir freilich noch viel zu ungentigend kennen. Erst eine genaue phonetische beschreibung der oberdeutschen mundarten mit sorgfältiger grenzbestimmung wird es uns vielleicht möglich machen den wirrwarr der alt- und mittelhochdeutschen schreibung aufzulösen und auch uber diesen punkt sicherer zu urteilen. Nach Weinhold steht $p$ in den heutigen mundarten nur in einigen wörtern, im bairischen, was sehr zu beachten ist, namentlich auch nach $m$, wo es auch mhd. geschrieben wird, während sonst im bairischen und elsässischen sogar spirans gesprochen wird. Daraus wird es doch sehr wahrscheinlich, dass wirklich ein 
phonetischer unterschied zwischen an- und inlaut im ahd. bestand, welcher auf dem schon vor der verschiebung vorhandenen beruhte. Die media wurde zur tenuis, die spirans zur media verschoben. Wenn auch in den inlaut $p$ eindrang, so widerspricht das, wie man auch dartiber urteilen mag, unserer auffassung nicht; denn auch für das aus $f$ erweichte $v$ tritt $p$ ein.

In der gutturalen reihe, zu der wir uns nunmehr wenden, sind die gotischem $g$ entsprechenden laute mit wenigen ausnahmen nicht im an- und inlaut durch die schrift unterschieden, wie diess bei den labialen im nordischen, angelsăchsischen und niederdeutschen der fall war, vielmehr ist fast therall ein und dasselbe zeichen $g$ in gebrauch. Aber schon die analogie macht es wahrscheinlich, dass auch hier unterschiede in der aussprache bestanden haben werden, welche so weit als möglich festzustellen unsere aufgabe ist. Wir werden, wenn wir im ausgedehnten masse die spirantische aussprache im inlaut vertreten finden, ein recht haben, diese unmittelbar an die von uns als wahrscheinlich ermittelte aussprache des gotischen anzukntipfen. Finden wir daneben den verschlusslaut, so lässt sich zum mindesten die möglichkeit, ja sogar die wahrscheinlichkeit, dass derselbe erst aus dem reibelaut entwickelt ist, in derselben weise dartun wie beim $b$. Unser hauptargument fur das gotische war, dass $g$ vielfach orst aus $h$ erweicht ist. Diese erweichung hat aber im allgemeinen gleichmåssig auch in den tubrigen dialekten stattgefunden. Allein im got. ist das $h$ erweicht nur in tagr. Dagegen ist sonst in den thrigen dialekten die erweichung weiter gegangen, z. b. in hahan preihan, fahan, juhiza, ahana, fraihnan, ganohs, pahan, vrohs, die tiberall ausser im got. $g$ zeigen. Bei andern erweichungen finden sich dialektische abweichungen. Im allgemeinen tbereinstimmend ist noch der sogenannte grammatische wechsel, der bei den verben der VII., VIII. und IX. classe stattfindet (z. b. ahd. ziuhu, zugumes) und den ableitungen aus denselben. Nur geht darin das nordische manchmal weiter z.b. in fleygja $=$ mhd. vlæhen, ebenso teygja, tiugari, leiga, leigja. Nur im nordischen erweicht ist elgr. = ahd. elaho, ags. elch. Speciell faeröisch sind faji (capio), tvaji (lavo). Nebeneinander stehen ags. altniederd. (psalmen) alth. svelgan nhd. schwelgen, und ahd. swelhan, 
mhd. swelhen. Manche erweichungen finden sich nur im ags. nd. und zum teil im md.; so ags. heagan acc. von heah, mnd. und md. hôgen, mnl. hoghen; ags. hêgan (exaltare), mnd. und md. irhogen; ags. scegon (viderunt), and. (psalmen) gesâgon, mnd. und md. sâgen, mnl. saghen, mnd. und md. geschâgen; alts. nigên; alts. (Freckenh.) tegotho (decimus), fries. tegotha, ags. preotegeota, seofonteogota (Benson), noch heute nd. tegede; md. negin, genegin = mhd. neehenen vgl. Ruckert a. a. 0. 57, noch jetzt im nd. noger compar. Speciell ags. sind fagan (varium), fägian (variare), fulgon praet. von felhan (aber auch got. fulgins, an. fialgr), sveger (doch daneben sveor und anderseits in den dazu gehörigen wörtern auch im hochdeutschen $g$ ). Dass die verwandlung des $h$ in $g$ nicht noch allgemeiner wurde, ebenso wie die des $f$ in $v$ oder $b$, lag jedenfalls daran, dass es sich zum blossen hauche verfltichtigte, und daher nur einfach ausfallen konnte. Jedenfalls mutssen alle so entstandenen $g$ einmal reibelaute gewesen sein. Finden wir diese zu verschlusslauten verschoben, so steht nichts im wege, dass durch dieselbe lautbewegung auch die thbrigen orst aus spiranten zu verschlusslauten geworden sind.

Es wird uns nun, denke ich, auch gelingen nachzuweisen dass die spirantische aussprache des $g$ im inlaut bei den verschiedenen germanischen stämmen entschieden tuberwiegt und bis in die älteste zeit zurtkckreicht. Dass der buchstabe $g$ zur bezeichnung derselben gebraucht wurde, darf uns deshalb nicht wunder nehmen, weil im lateinischen alphabet kein anderes verwendbares zeichen vorhanden war. $i$ wurde zur bezeichnung des vokales und der halbvokalischen palatalen spirans gebraucht welche in ältester zeit wol viel mehr als vokal wie als consonant empfunden wurde (vgl. oben s. $158 \mathrm{anm}$.) Dem gutturalen spiranten lag denn doch wol der homorgane verschlusslaut näher. Auch wurde ja das $g$ bereits im romanischen vor hellen vokalen als spirans ausgesprochen. Nichtsdestoweniger wechselt vielfach $g$ mit $j$. Seltener ist der fall, dass $j$ fur $g$ gebraucht wird, sehr häufig aber im hoch- und niederdeutschen und im ags. das umgekehrte, so dass es keines beleges bedarf, was vielleicht als ein zeugnis dafur anzusehen ist, dass das $j$ sein vokalisches element zu verlieren beginnt. *) Dieser wechsel

*) In der häufigen schreibung ig erscheinen beide elemente deutlich gesondert, gerade wie in der schreibung $u u, u b$. Doch wird auch in der 
zeigt, dass das $g$ wenigstens an bestimmten stellen oine dem $j$ nicht zu fern liegende aussprache gehabt haben muss.

Aus dem altnordischen lassen sich mehrere momente anfuhren, die dafür sprechen, dass $g$ im in- und auslaut spirant war. Ueberall, wo es ursprtinglich im auslaute stand, ist es geschwunden mit verlängerung des voraufgehenden rokals, wenn er kurz war. Es ist also ganz analog behandelt wie $h$, wozu es wahrscheinlich vorher nach verlust des stimmtones geworden war. Die ausnahmen von dieser regel im imp. und im nom. der neutra erklären sich vielleicht daraus, dass das vokalische auslautgesetz hier später in kraft getreten ist als in den tubrigen germanischen sprachen, wofür ja auch die altesten runeninschriften und die gestalt der aus dem nordischen entlehnten wörter im finnischen sprechen. In der verbindung $g j$ nach langem vokal fällt $g$ öfter aus z. b. in teyja für teywja, und daher wird dann umgekehrt oft $g j$ statt des richtigen $j$ geschrieben vgl. Holtzm. 108. Wir haben darin offenbar éinen beweis für die bereits eingetretene palatalisierung zu sehen. Die gutturale spirans ist durch das folgende $\rho$ in die palatale verwandelt. In stî $a$ und ôask ist das $g$ zwischen den vokalen ausgefallen gerade wie sonst $h$. $\mathrm{Zu}$ beachten ist auch die in dem Gutalagh und auch sonst vorkommende schreibung $g h$. Die aussprache des neuisländischen und dänischen spricht tlbereinstimmend fur verschlusslaut im anlaut und reibelaut im inund auslaut. Im isländischen wird anlautend vor harten vokalen $g$, vor weichen $g j$ gesprochen, in- und auslautend vor harten gutturaler reibelaut, vor weichen palataler $=j$. Im dänischen ist $g$ im anlaut immer verschlusslaut. Im in - und auslaut iat es entweder gutturaler oder palataler reibelaut, oder wird zu $i$ vokalisiert, oder verklingt ganz, oder endlich wird $z u v$, welches dann wider teilweise zu $u$ vokalisiert wird. Dem letztgenannten tubergange werden wir auch in andern dialekten, namentlich im englischen begegnen, eben so wie dem umgekehrten von $v$ in $g$. Wir sind gewis uberall berechtigt diesen wechsel als ein zeugnis fur die aussprache des $g$ als spirans

aussprache eine solche zerteilung stattgefunden haben, gerade wie man in heutigen mundarten deutlich nazijon $=$ frz. nation oder spijon $=$ spion hört. 
anzusehen. Er vergleicht sich zunăchst mit dem wechsel von $j$ und $w$. Ueberhaupt sind die spiranten, tönende wie tonlose, vielmehr dem wechsel unter einander ausgesetzt als die verschlusslaute. Aus der dänischen aussprache lässt sich allerdings kein sicherer schluss auf die alteste zeit machen, da auch das aus $k$ erweichte $g$ zur spirans wird. Wir mitssen uns also namentlich auf die neuisländische aussprache und die aus dem an. selbst beigebrachten anzeichen stlitzen und können nur constatieren, dass das dänische dazu stimmt. Doch lässt sich wenigstens eine spur von dem thbergange des $g$ in $v$ schon in alter zeit nachweisen in dem von Grimm aus Saxo citierten Svipdavus. In bestimmten fällen steht der explosivlaut. Wenn im neuisländischen $g$ vor $n, l, \delta, g$ mit verschluss gesprochen wird, so ist diess vollkommen analog der aussprache des $f$ vor denselben consonanten als $b$ und erweist sich dadurch als eine jthngere veränderung. Dazu kommt, dass auch hier $g h$ geschrieben wird, und dass in der skaldenpoesie dieses $g$ auf $g$ zwischen vokalen reimt (vgl. Gislason oldnordisk formlære §81). Ebenso ist die heutige aussprache nach consonanten erst jung. Auch hier wird in älterer zeit $g h$ geschrieben (vgl. ebendas.) und im dänischen spirant gesprochen, wenn das $g$ nicht ganz verstummt. Sicher ist $g$ schon im an. wie in den neuern sprachen verschlusslaut nach $n$ und in der gemination. Fur den ersten fall folgt diess daraus, dass $n g$ im auslaut wie $n k$ in $k k$ thergeht. Ftir geminiertes $g$ findet sich $k k$ geschrieben (Holtzm. 106). Wenn in der dänischen aussprache $g$ vor $s$ und $t$ zu $k$ wird, so ist anzunehmen, dass diess aus dem zunächst tonlos gewordenen spiranten entstanden ist, da auch altes $h$ vor den selben consonanten zu $k$ wird, vor $t$ nur in wörtern die aus dem deutschen entlehnt sind, da es in echt altnordischen geschwunden war, z. b. magt, sprich makt. Spuren dieses tberganges finden sich schon im an., indem zuweilen $k s$ fur gs geschrieben wird. Das neutrum -likt von den adjectiven auf ligr kommt nicht in betracht, da bei diesen $g$ erst aus $k$ erweicht ist. Der tubergang von $h$ vor $s$ in $k$ ist wol uberhaupt in sämmtlichen neuern germanischen dialekten eingetreten. Ihm vergleicht sich auch das im an. zuweilen vorkommende $p s$ fur $f s$ (Holtzm. 116). Auch fur den von $h t$ in $k t$ werden wir in deutschen mundarten analogien finden. Und vielleicht ist auch das 
an $p t$ fur $f t$, welches auch in gotischen eigennamen und in den merseburger sprichen vorkommt so aufzufassen, dass wirklich $p$ erst aus $f$ entstanden ist. Ein anderer fall, in dem $g \mathrm{zu} k$ wird, ist hinter $t$ und $s$. Zum teil ist dabei $g$ der anlaut des zweiten teiles eines compositums; aber es wird auch z.b. amatki aus âmattigi. Wir werden auch hier keinen anstoss daran nehmen, dass das $k$ aus einem spiranten entstanden ist. Wir werden demselben ubergange bei $\operatorname{dem} b$ wider begegnen. So entsteht auch huspreyja aus hissfreyja. Inlautender verschlusslaut steht im altn. auch vor $j$, wo $g g$ geschrieben wird, welches aber hier wie Holtzmann 108 ausgefuhrt hat, nicht gemination bezeichnen kann. Dass $g g$ zur bezeichnung des verchlusslautes angewendet wird, beweist einerseits, dass derselbe in der gemination gesprochen wurde, anderseits, dass diess sonst nicht der fall war und daher zur unterscheidung von der gewöhnlichen aussprache in ermangelung eines besseren die verdoppelung zu hulfe genommen werden muste. Wenn so das nordische, neuisländische und dänische für die ursprïnglichkeit der spirans im inlaut spricht, so haben wir wol ein recht den verschlusslaut im schwedischen für unursprtinglich zu halten, welcher hier nach den angaben der grammatiken uberall steht ausser noch $r$ und $l$, wo $j$ gesprochen wird.

Für das ags. lässt sich die spirantische natur des inlautenden $g$ noch mit grösserer sicherheit dartun. Schon dass hier die verwendung des $g$ für $j$ besonders häufig ist, weist darauf hin, dass es wenigstens zum teil nicht bedeutend verschieden davon geklungen haben kann. Im neuags. und altengl. wird es im inlaut ganz verdrängt von dem neuerfundenen zeichen $z(g h)$. Da dieses sonst auch fur altes $j$ und furr $h$ im auslaut und vor $t$ gebraucht wird, so kann man daraus den schluss ziehen, dass es eine spirans, und zwar gutturale wie palatale, tönєnde wie tonlose bezeichnet. Ueberblicken wir nun die wandlungen des in- und auslautenden $g$ im ags. und engl. Im auslaut geht $g$ im ags. und auch im neuags. nach langem vokal und nach consonanten gewöhnlich, seltener nach kurzem vokal in $h$ uber (Holtzm. 210. Koch $\S 177$ ), wofür vom neuags. an wie sonst für auslautendes $h$ auch $g h$ eintritt. Da wir darin jedenfalls wider den bekannten verlust des stimmtones zu sehen haben, so muss auch im inlaut spirans bestanden haben. 
Dieselbe verwandlung tritt ein vor $s$ und $\partial$ bei syncope des vokals z. b. bȳhst, bȳh $\delta$ aus bugan. In schwachen verben wird $g$ verwechselt mit dem $j$ des suffixes. Es tritt daher einerseits fur dieses ein z. b. in freogan (amare Holtzm. 212), anderseits geht, wenn die wurzel ursprïnglich $g$ hat, nicht bloss das $j$, sondern auch das damit verschmolzene $g$ verloren $z$. b. eavan yvan aus augjan (Holtzm. 210). Häufig ist der wechsel zwischen $g$ und $v$, der ebenso $\mathrm{zu}$ beurteilen ist wie im dänischen. Auf der einen seite entsteht im ags. häufig $g$ aus $v$ (Holtzm. 211) z. b. grag, engl. gray. Auf der anderen geht im neuags. nicht selten $g$ in $n$ uber, welches dann später vokalisiert wird (Koch § 178). Ganz analog tritt im auslaute statt des $g h$ in der aussprache ein $f$ ein. Die gewöhnlichste verwandlung des $g$ ist, dass es zu $i$ vokalisiert wird. Die erste stufe dazu findet sich wol angedeutet in der in den northumbrischen evangelien vorkommenden schreibung weig für weg u. a.m., bei Lazamon weize, faeizer etc. Das vorgesetzte $i$ bezeichent wol den ubergang in die palatale, auch wol schon halbvokalische spirans, gerade wie im altn. die schreibung seigja, von wo aus dann der übergang in den blossen vokal leicht ist. Es findet sich auch in den evangelien und ist bei Lazamon das gewöhnliche, bei dem man statt altem age oder ag findet $a i, c e i, e i, e$. Merkwürdiger weise findet sich gerade die letzte schreibung, nach der das $g$ einfach verloren zu sein scheint, häufig schon im altags. (Holtzm. 209). ig und ig werden naturlich zu $i$ zusammengezogen z. b. stîrâp, älmihti. Verschlusslaut wurde im ags. gesprochen wie im altn. nach $n$ und in der gemination. Niemals geht nach dem $n$ das $g$ auslautend in $h$ tuber. Im neuengl. bleibt der verschlusslaut oder es tritt in der aussprache dsch ein, welches in deutschen worten immer aus einem verschlusslaute hervorgeht. Fur die gemination ist schon die schreibung $\mathrm{cg}$ beweisend, welche zugleich ein zeugnis für das bedürfnis nach scheidung von dem gewöhnlichen $g$ ist. Im neuengl. bleibt wider entweder $g$ oder wird $d g$ (dsch). Nur in den verben licgan, lecgan, secgan, bycgan tritt vokalisation ein, aber später als bei dem einfachen $g$; noch im mittelengl. ist sie nicht allgemein durchgedrungen. Daher ist sie auch nicht mit der des einfachen lautes auf eine stufe zu stellen. Bei den drei ersten verben kann ubrigens die analogie des praeteritums mitgewirkt haben. Der ursprungliche unter- 
schied der aussprache des einfachen und des verdoppelten $g$ ist also klar. Ebenso wird auch in den romanischen wörtern das $g$ nicht vokalisiert oder ausgestossen, sondern es bleibt vor consonanten und harten vokalen als $g$ z. b. legal, tigre, vor weichen vokalen als $d s c h$, wie es schon im franz. gesprochen wurde. Wenn Koch § 179 ausfall des romanischen $g$ im engl. hehauptet so verlegt or lauttibergänge in diese sprache, welche die angeftihrten wörter schon vor ihrer aufnahme in dieselbe durchgemacht haben. Wir sehen also auch hieraus, dass nicht der verschlusslaut von der vokalisation betroffen wird. In einigen wenigen wörtern hat sich im neuengl. einfaches $g$ im auslaut nach vokalen erhalten und wird mit verschluss gesprochen: whig (daneben whey), twig, egg, drag, hag, crag, beg, nag (Koch $\S 178$ ). Wir werden keinen anstand nehmen in diesen vereinzelten fällen die entstehung des $g$ aus der spirans für wahrscheinlich zu halten, zumal da das $g$ von ags. twig und äg erst aus $j$ hervorgegangen ist. Uebergänge der auslautenden spirans in den verschlusslaut lassen sich auch sonst im neuengl. nachweisen. Aus ags. eolh wird elc; gh, welches aus ags. $h$ hervorgegangen ist und im altengl. sicher die harte gutturale spirans bezeichente, wird $k$ gesprochen in lough (see), shough (pudel) und hough (kniekehle); in letzterem worte schwankt die aussprache, indem daneben mit dem bekannten wechsel des organs $f$ gesprochen wird. In hiccough (schucken) hat dieser wechsel durchaus stattgefunden und ist dann das $f$ meistens in der aussprache in $p$ thbergegangen, so dass nun auch daneben hiccup geschrieben wird. Alles diess sind vollkommen unanfechtbare lauttbergånge, nach deren analogie wir auch die auslautenden $g \mathrm{zu}$ beurteilen berechtigt sind.

Auch im anlaut muss $g$ im ags, zum teil als spirant gesprochen sein. Dafür spricht schon die gerade hier häufige verwendung des zeichens $g$ für $j$ vor hellen vokalen, welche in manchen wörtern ausnahmslos statt hat. Vor harten wird ge für $j$ geschrieben. Das $e$ dient also dazu anzudeuten, dass $g$ als spirans zu sprechen ist. Wir werden ihm daher dieselbe bedeutung zuschreiben, wenn es vor $a$ hinter altem $g$ geschrieben wird auch in fällen, wo nach den sonstigen lautgesetzen kein ea eintritt z. b. geat, geâton. Auch dient ja vollkommen analog die schreibung sce zur bezeichnung des reibelautes. Im neuags. 
werden viele wörter im anlaut mit $\zeta$ geschrieben. Die partikel ge vokalisiert sich sogar $\mathrm{zu} i$, welches im neuengl. mit wenigen ausnahmen ganz abfällt. Aber im neuengl. haben nur ein paar wörter $y$, alle tibrigen mit verschluss gebildetes $g$. Diese tatsachen nötigen uns anzunehmen, dass diess $g$ mindestens zum teil erst aus dem reibelaut entstanden ist, den wir, soweit unsere quellen zurïckreichen, als das älteste ansetzen mulssen.

Im niederdeutschen und einem teile des mitteldeutschen wird heute ausser nach $n$ im inlaut tönender, im auslaut tonloser spirant gesprochen. Für das alter dieser aussprache lassen sich schon aus den ältesten quellen beweise beibringen. Im Heliand findet sich auslautend ofter $h$ geschrieben wie im ags. (Holtzm. 150), woneben das einmalige ôdoc im Mon. nicht sehr in betracht kommen kann. In der Essener rolle findet sich ahtodoch, viftech. Sonst bleibt allerdings $g$ in den ältesten denkmalen, wol aus demselben grunde wie im gotischen. Umgekehrt findet sich $g$ für auslautendes $h$ im Hel. in noh wie häufig in späteren nieder- und mitteldeutschen quellen, nur erklärlich dadurch, dass $g$ als spirans, und zwar im auslaut als tonlose spirans gesprochen wurde. Ferner fällt $g$ in dem suffix ig im Cott. öfter ab wie das $h$ und tritt umgekehrt an auslautendes $i$ an wie gibithig. Schreibungen wie toiu statt togiu im Mon. und boi statt bogi in gl. Arg. sind gewis eben so aufzufassen wie die von uns besprochenen analogen fälle im an. und ags. (teyja für teygja etc.). Verwandlung des $w$ in $g$ findet sich wenigstens in niguni. Vollkommen klar ist das verhältnis in jüngerer zeit. In den altfriesischen rechtsquellen ist der ubergang von auslautendem $g$ in ch durch die schrift fast durchgängig bezeichnet. Im inlaut spricht' die häufige vokalisierung fur dio spirantische aussprache. Dagegen tritt in der gemination und nach $n$ palatalisierung resp. dentalisierung ein wie im anlaute, welche den verschlusslaut voraussetzt. Im mnd. mnl. und mittelfränkischen ist ebenfalls $c h \mathrm{im}$ auslaut und im inlaut vor harten consonanten die gewöhnliche schreibung und die reime beweisen das zusammenfallen rait got. $h$ und in dem letztgenannten dialekte auch mit got. $k$ nach vokalen. Oft wird $g$ geschrieben, aber eben so gut auch für got. $h$. Auch in das studfränkische und hessische hinein reicht die verwandelung des auslautenden $g$ in $c h$ z. b. bei Herbort und in Hartmanns Credo, 
während z.b. in der Elisabeth häufig $c$ im auslaute geschrieben wird und keine reime auf ch sich finden. Fur das mnd. ist noch die schreibung gh zu beachten, die auch sonst vorkommt und doch wol analog dem ch die spirans bezeichnen soll. Allerdings wird auch $n g h$ und $g g h$ geschrieben, während in diesen verbindungen nach analogie der tubrigen dialekte der verschlusslaut erwartet wird und auch im auslaut $n c$, nicht $n c h$ eintritt. Auch in der heutigen aussprache des niederdeutschen bleibt $n g$, resp. gutturaler nasal und im auslaut $n k ; g g$ ist zu $k k$ verschoben, nur in schwachen verben, wo die verdoppelung durch ein $j$ bewirkt war, ist wider die spirans eingetreten: lejjen, seijen.

Auch im anlaut lassen sich von alter zeit her spuren spirantischer aussprache erkennen. Aus dem Heliand lässt sich allerdings nichts weiter dafür anfuhren, ausser dass $g$ vor hellen und $g i$ vor dunkeln vokalen für $j$ gebraucht wird. Aber in der ubersetzung der predigt des Beda findet sich jegivan, in den Merseb. gl. jernihed. Im afries. ist $j$ für $g$ vor $e$ nicht selten; ja die vorsilbe ge wird zu $e$ oder $i$ zusammengezogen, wofür sich auch ein beispiel in den Mers. gl. findet. Noch in ein paar andern wörtern fällt $g$ im afries. ab (unge, ungath, iuth). Heute ist im niederdeutschen uberall die partikel ge zu einem schwachen $e$ geworden oder ganz abgefallen. Im mnl. wird auch im anlaut vor hellen vokalen $g h$ geschrieben. Eine spur, dass vor ursprünglich harten vokalen reibelaut gesprochen wäre wie im ags. finde ich nicht, doch lässt sich auch das gegenteil nicht erweisen. Heute unterscheiden sich die niederdeutschen mundarten in der aussprache des anlautenden $g$. Es stehen neben einander verschlusslaut, gutturaler und palataler spirant, so dass wol der erste das hïufigste, der zweite das seltenste ist. Von dem palatalen spiranten ist es klar, dass er erst aus dem gutturalen entstanden sein muss; aber ob dieser oder der rerschlusslaut altertumlicher ist, wird schwer $\mathrm{zu}$ entscheiden sein.

Für das hochdeutsche gilt dasselbe, was bei den labialen bemerkt ist. Wo wir im in- und auslaut den verschlusslaut finden, kann or nach einem gesetze, das notwendig einmal wirksam gewesen sein muss, aus der spirans entstanden sein. Aber auch in Oberdeutschland stossen wir auf den spiranten. 
Allerdings findet sich besonders in den ältesten quellen $k$. Aber im ganzen uberwiegt $g$ bedeutend im inlaute auch in denkmälern, die im anlaut in der regel $k$ haben. Im auslaut erscheint schon von fruhester zeit $c h$ neben $g$ und $c$ oder $k$. In vielen mhd. handschriften erscheint es fast regelmässig. Reime auf $c h=$ got. $h$ oder $k$ sind nicht gerade häufig, kommen aber auch bei guten dichtern wie Hartmann von Aue vor. Ich habe (mhd. schriftspr, p. 26.) die ansicht ausgesprochen, dass diess oberdeutsche ch ganz anders aufzufassen sei, als das niederdeutsche und durch eine doppelte verschiebung zu erklären. Ich trage jetzt bedenken daran festzuhalten, da auch andere gründe dafur sprechen, dass inlautendes $g$ mindestens in bestimmten teilen von Oberdeutschland als spirans gesprochen wurde, so dass ihm also ch dem auslautgesetze gemäss unmittelbar entsprach. Allerdings sind zwei bedenken dabei einmal dass oft auch $n c h$ geschrieben wird, während $g$ nach $n$ sicher explosivlaut war, und dann, dass dies ch gerade in gegenden zu herschen scheint, welche in der verschiebung am weitesten gehen, während in einem grossen teile von Mitteldeutschland $c$ neben $g$ in der schreibung therwiegt und in den reimen keine vermischung mit $c h$, wol aber mit $k=$ got. $k$ vorkommt. Fur inlautenden spiranten spricht sonst noch folgendes: Hăufig wird $g$ fur $j$ geschrieben und dieses $g$ reimt nicht selten auf echtes $g$ (Weinh. al. gram. 215. bair. gram. 178). Es bleibt uns nur die wahl zwischen zwei möglichen annahmen. Entweder wurde das $g$ wie $j$ gesprochen, oder $j$ ist in $g$ thergegangen. Ist letzteres der fall, so haben wir wider einen beweis fur den ubergang der spirans in den verschlusslaut. Wir sind hier in einer ahnlichen ungewisheit wio b9i $b$ und $w$. Im allgemeinen scheint mir die erstere annahme das wahrscheinlichere. Aber zum teil mag auch thergang des $j$ in $g$ erfolgt sein, namentlich nach $r$, wo das $g$ auch in der neuhochdeutschen schriftsprache sich findet. Auch im anlaut findet sich in neueren mundarten $g$ fur $j$, z. b. in der Oberpfalz und im Voigtlande. Vokalisierung des $g$ ist auch in Oberdeutschland häufig, besonders vor $t$ (gramm. I 2426). Wenn age, ege zu ei werden, so kann das wol nicht so aufgefasst werden, dass das $g$ ausgestossen und dann $a-e$ oder $e-e$ zu $e i$ zusammengezogen wäre. Wir müsten dann wenigstens uberall $i$ statt des stummen $e$ haben. Nun 
aber ist das $e$ nur in der kleineren anzahl der fälle aus $i$ geschwächt und die zusammenziehung ist auch erst eingetreten nachdem die schwächung erfolgt ist. Das $i$ des diphthongs ist vielmehr aus dem zunächst $\mathrm{zu} j$ geworden€n $g$ entstanden. Viel weiter in diesen zusammenziehungen, ähnlich wie das englische und friesische gehen die östlichen mitteldeutschen mundarten vgl. Weinh. schles. mundarten 47.64. Im schlesischen findet sich z. b. auch moi für mag, wo an eine zusammenziehung aus $a-e$ gar nicht zu denken ist. Uebergang von $w$ in $g$, zum teil durch den reim bestätigt, findet sich besonders im elsässischen (alem. gr. 216). Ueber das vorkommen desselben in Mitteldeutschland vgl. Rückert a. a. o. 57. und Pfeiffer, Jerosch. L. XVII.

Heute wird die spirans gesprochen im Elsass, in Niederschwaben, der Oberpfalz, Niederöstreich und teilen von Tyrol. Es scheint also, dass sie im verhältnis zum mhd., wo sie im auslaut so uberaus häufig durch $c h$ bezeichnet wird, an umfang eingebusst hat. In Mitteldeutschland tiberwiegt sie. Es ist mir unmöglich ihre verbreitung genau anzugeben. In Schlesien und und dem grösseren östlichen und südlichen teile von Obersachsen wird verschlusslaut gesprochen, im inlaut tönender, im auslaut nach den mir etwas zweifelhaft erscheinenden angaben von Weinhold und Rückert in Schlesien wenigstens nach langem vokal gleichfalls tönender, in Sachsen tonloser. Ausgenommen ist davon die endung - igh und sontigh, montigh, herbrigh. Dass auch hier der verschlusslaut junger ist, wird dadurch wahrscheinlich, dass derselbe im schlesischen auch aus altem $h$ entsteht, z. b. flog (fugit), sag (vidit), sickt, erwackt, versmacktet, formen die schon bei Opitz und Gryphius vorkommen (Weinh. schles, mundarten 84, 86), Aus dem angrenzenden obersăchsischen gebiete kenne ich sak. Auch weist der dem abfall des $h$ analoge abfall des $g$ in anderen wörtern (Weinh. 84.) und die gerade hier häufige vokalisierung des $g$ auf ältere spirantische aussprache hin.

Mit der betrachtung der dentalen betreten wir das am meisten streitige gebiet. Ich führe zunächst zwei punkte an, welche entscheidend dafür sind, dass $b$ in der ältesten zeit, bis zu der wir zurückgehen können, die gewöhnlich geläugnete geltung einer spirans hatte. Erstens entsteht in einigen wör- 
tern aus älterem $b$ eine andere spirans. So $h$ in got. mabl, mapljan, ags. mäðel, mäðelian, ahd. madul in eigennamen $=$ ahd. mahal, mahaljan, as. mahal, mahlian, an. mit regelrechtem ausfall des $h$ und contraktion mâl, moela. Mehr beispiele gibt es für den ubergang in $f$ : got. pliuhan $=$ ahd. as. fliohan, ags. fleon, an. flyja; got. blaihan = ahd. flêhan, flêhôn, as. giflihian (gifťhid Hel. 1460); ahd. dinstar, md. dinster, as. thiustri (mit vokalisierung des nasalklanges), ags. peostri, pysstre $=$ ahd. finstar; got. aipbau, ahd. eddo, odo, ags. oðঠe = as. ef $a$, ef內o, fries. ieftha; ${ }^{*}$ an. pengill, ags. pengel $=$ ags. fengel; im an. gehen neben einander bili und fiol. Wie liesse sich das erklären, wenn $b$ aspirata oder auch affricata gewesen wäre? Wir haben vielmehr in diesen ubergängen wider beispiele des wechsels der spiranten unter einander. $\mathrm{Zu}$ vergleichen ist damit der ubergang des $h$ in $f$, der gleichfalls in alter zeit stattgefunden hat (vgl. J. Schmidt, z. indogerm. vokalismus s. 53.) und im englischen (Koch 182), und der im niederdeutschen häufige umgekehrte des $f$ vor $t$ in $c h$. Ueber den wechsel der tönenden spiranten ist oben $\mathrm{s.} 175 \mathrm{f}$. gesprochen. Die wandelung von dentalem $s$ ( $s^{4}$ nach Brücke), als welches wir $b$ auffassen, ist bekanntlich einer der leichtesten lautubergänge, den man sich denken kann. Man braucht nur die zunge und die beim $s^{4}$ vorgeschobene unterlippe etwas zuruckzuziehen, so dass die letztere der obern zahnreihe genähert wird. Bekannt ist ja auch das vorkommen dieses uberganges im lateinischen und russischen. Wenn auch im äolischen $\vartheta$ in $\varphi$ tubergeht, so muss man wol daraus schliessen, dass in diesem dialekt die aspiraten bereits zu spiranten geworden waren.

Der zweite punkt, den ich meine, ist folgendes: Im alts. und ags., auch im an. zum teil, schwindet vor $p$, das dann auch zu $\partial$ erweicht erscheint, der nasal z. b. alts. ô $\partial a r$ aus got. anpar. Dieser schwund des nasales tritt sonst noch ein vor $s$ und $f$; vor $h$ ist er bereits in einer fruheren periode in allen germarischen dialekten eingetreten. Nirgends zeigt er sich vor verschlusslauten. Wir werden daher auch $b$ nicht zu den letzteren rechnen, und es ergibt sich dann die einfache regel: der nasal schwindet vor den harten spiranten.

•) Oder ist vielleicht letzteres die ältere form? 
Diese beiden argumente, das verhältnis im gotischen, die analogie der beiden andern consonantenreihen müssen uns fur die bcurteilung der entwickelung des $\beta$ massgebend sein. Im an. sind im anlaut $d$ und $p$ in gleicher wcise geschieden wie im got. Inlautend sind sie nur nach $l$ und $n$ geschieden: got. $l p, n p=$ an $l l, n n$; got $l d, n d=$ an. $l \delta$, n內 oder $l d, n d$. In jeder andern stellung sind sie zusammengefallen: in der gemination steht $d d$; sonst steht in den ältesten handschriften $p$, wofür später $\partial$ eintritt, welches selten auch im anlaut geschrieben wird. Wir sehen, das ursprüngliche verhältnis ist ganz so wie bei den labialen und die gröste wahrscheinlichkeit spricht daher dafür, dass es gerade so aufgefasst werden muss. Der hauptgrund, weswegen man sich dagegen sträubt, ist, dass im schwedischen, dünischen und faerœischen anlautend für $b$ der explosivlaut steht, im schwedischen und bei vielen consonantenverbindungen in allen neunordischen sprachen, ja selbst im jüngeren an. auch inlautend. Dazu kommt der im inlaut sehr seltene, im auslaut häufigere wechsel von $\delta$ und $t$ (Gislason 118, 4.5). Man nimmt daher an, dass ursprünglich noch aspirata gesprochen sei, die anlautend nur im isländischen zur spirans geworden, während in den andern sprachen der hauch abgefallen sei. Ich will einstweilen davon absehen, ob wirklich bei dieser auffassung die entstehung der neunordischen $t$ und $d$ aus $p$ vom physiologischen standpunkte aus begreiflicher wird. Aber wie erklärt man sich das zusammenfallen mit got. $d$ ? Wir können doch nicht dem in ältester zeit gleichmässig geschriebenen $\boldsymbol{b}$ eine so ganz verschiedene geltung zuschreiben, dass es bald einen doppellaut, die aspirata, bald einfache spirans oder etwa tönenden verschlusslaut bezeichent hätte? Und wie hätte aus der grot. media, mag man sie nun als spirans oder als verschlusslaut auffassen, im in - und auslaut aspirata werden sollen, um später wider zu tönendem reibe- oder verschlusslaut zu werden? Die auslautenden $t$ für $\partial$, woneben umgekehrt $\succsim$ für $t$ rorkommt, beruhen wol darauf, dass das auslautende $t$ schon wie im neuisländischen zur spirans geworden war. Die paar fälle im inlaut können verschreibungen sein; wenn sie einen lautlichen grund haben, so können sie nur ein anzeichen sein, dass die ursprüngliche spirans sich bisweilen dem explosivlaute näherte. Auf der andern seite findet sich 
auch $z$ für $\partial$. Ferner geht in cinigen seltenen fällen $\partial$ wie $s$ in $r$ uber, z. b. bar (orabat) und namentlich in der 2. pers. pl. (Gislason 118, 6), weshalb Gislason mit grosser wahrscheinlichkeit vermutet, dass auch in der 3. pers. sing. das $r$ auf dieselbe weise zu erklären sei. Spuren dieses überganges finden sich auch im neuisländischen (ib. anm.). Wie ferner $f$ aus $p$ entsteht, so umgekehrt $p(\partial)$ aus $f$ in fidrildi aus fifril $i$ und pjosir für älteres fjosir. Ebenso haben wir den bekannten spirantenwechsel darin $\mathrm{zu}$ sehen, wenn zuweilen $g \mathrm{zu} \partial$ oder $\delta \mathrm{zu} g$ wird (Gislason 118, 2). Die erweichung im in - und auslaut war bei aspiraten nicht möglich; sie beruht vielmehr auf der allgemeinen neigung der spiranten dazu wie die des $f$ und $h$. Die erweichung ist schon vor der zeit, aus der wir uberlieferungen haben, vor sich gegangen, wiewol sie durch die schrift nicht bezeichnet wird. Das geht daraus hervor, dass $p$, zuu $d d$ geworden ist und dass in consonantenverbindungen frühzeitig $d$ für $b$ eintritt. Daher ist es auch so wenig wie bei den labialen nötig cine verhärtung der gotischen media anzunehmen. Diese verhältnisse sind meiner ansicht nach so klar, dass wir uns entschliessen müssen da, wo der verschlusslaut erscheint, anzunehmen, dass er aus der spirans entstanden ist. Diese entstehung können wir im inlaut in den an. denkmälern verfolgen. In den ältesten quellen kommt nach $n$ und $l$ noch $\partial$ (b) vor, nach $m$ ist es die regel; bald wird $d$ herschend; ebenso nach $m b, l f, l g$, ng. Nach $p, k, s$ steht $t$, aber in den alteren denkmälern nach $p$ und $k$ gewöhnlich, nach $s$ wenigstens noch in runeninschriften $\partial(p)$. Es ist hier zugleich verhärtung eingetreten durch assimilation an den vorhergehenden consonanten nach ausstossung des ursprünglich dazwischen stehenden vokales. Ebenso wie inlautendes $p$ oder $\partial$ wird das des enklitisctien $b u$ behandelt. Auch vor $t$ wird $\partial \mathrm{zu} \mathrm{t}$, was um so weniger gegen ursprtinglich spirantische aussprache beweisen kann, weil $t t$ auch aus st entsteht und ebenso $d d$ aus $z d$. Wenn nun in den neueren sprachen auch im anlaut die spirans in den explosivlaut tibertritt, so ist das dem inlaut vollkommen analog. Wir können auch in anderen fällen bemerken, dass anlautende consonanten gerade so behandelt werden wie inlautende nach anderen consonanten. Im færœischen tritt überall $t$ ein, im schwedischen und dänischen im pron. der 2. pers. 
und im artikel nebst den ableitungen davon, $d$, sonst $t$. Da auch gerade in diesen wörtern im an. am öftesten $\partial$ geschrieben wird und das englische dazu stimmt, so wird die erweicliung ir den dem dän. und schwed. zu grunde liegenden dialekten alt sein, während sie im færœischen und isl. nicht eintrat.

Wälırend im nordischen got. $p$ und $d$ in in - und auslaute zusammengefallen sind, sind sie in den ältesten quellen der südgermanischen dialekte geschieden, indem ersterem $b$ oder $\partial$, letzterem $d$, hochdeutsch zu $t$ verschoben, entspricht. Wir müssen nach der analogie der beiden anderen consonantenreihen und gemäss dem von uns erkannten lautwert des gotischen annehmen, dass dieses ohne zweifel mit verschluss gesprochene $d$ erst aus der spirans verschoben ist. Der unterschied von der entwickelung der labialen und gutturalen besteht nur darin, dass bei diesen die verschiebung zum verschlusslaut auf ein kleineres gebiet eingeschränkt ist. Ein teil der $d$ war schon im got. aus $b$ entstanden, im sudgermanischen hat sich die anzahl derselben bedeutend vermehrt. In diesen fällen muss erweichung des $p$ eingetreten sein, bevor die verschiebung zum verschlusslaute erfolgte. 'Trat sie später ein, so blieb zunächst der reibelaut. Wie bei den labialen und gutturalen finden sich einzelue abweichungen der dialekte unter einander, grammatischer wechsel, schwankungen in einem und demselben dialekt. $\mathrm{Nur}$ im got. ist erweichung eingetreten in skaidan as. scêthan, ahd. sceidan. $\mathrm{Ob}$ im an. einzelne $b$ fruher tönend geworden sind, ehe die allgemeine erweichung eintrat, lässt sich nicht entscheiden ausser bei den verbindungen $n p$ und $l p$. Letzteres ist im sudgermanischen durchweg zu $l d$ geworden, während es im an. als $l l$ von $l d$ geschieden ist. Nur aldr, öld ist abweichend von got. alpeis, daneben aber steht noch elli. $n p$ und $n d$ bleiben auch im sudgermanischen geschieden, indem von ersterem im as. und ags. der nasal schwirdet; doch schwankt es nach und ein solches schwanken findet auch im an. statt in fintrict und finda. $\quad b b$, welches im an. allgemein zu $d d$ wird, bleibt im ags., abweichend auch von der analogie der labialen und guttaralen, $b l$ oder $\partial \delta$; ebenso im as. in ettha; verdoppelung durch folgendes $j$ tritt im as. nicht ein; in queddan ist wol schon vor der verdoppelung $d$ eingetreten wie in quidi. 
Betrachten wir nun die entwickelung der zunächst nicht zum verschlusslaut verschobenen $p$ und $\partial$. Im ags. werden beide zeichen unterschiedslos gebraucht, im nags. kommt $\partial$ allmählich ausser gebrauch, im mengl. tritt allgemein th ein. Der gebrauch des zeichens $\partial$ weist auf das vorhandensein tönender aussprache hin, wenn auch die schreiber den unterschied nicht richtig durchzuführen vermochten Dass dieselbe im inlaut im ags. wie im neuengl. allgemein war, zeigt besonders der häufige wechsel mit $d$, das sowol für $\partial$ eintritt, als umgekehrt dieses für $d$. In letzterem falle könnte man die $\partial$ zum teil als unverschobene reste der alten spirans ansehen; aber sie treten auch in lateinischen wörtern für $d$ ein und in einigen wörtern entsteht erst im neuengl. th aus $d$, so dass das vorkommen der verwandlung des verschlusslautes in die spirans keinem zweifel unterliegt. Als beweis dafür, dass die erweichung schon in alter zeit stattgefunden haben muss, lässt sich auch noch anfuhren, dass dieselbe in den lehnwörtern aus dem griechischen nicht eintritt, weil die neigung dazu vorüber war. Im anlaut kennt die heutige schriftsprache die erweichung nur in den pronominalstämmen. Da aber in vielen mundarten $t h$ in $d$ übergeht, so ist es nicht unwahrscheinlich, dass schon in alter zeit auch in anderen wörtern das $b$ zum teil tönend geworden war.

In der heutigen aussprache auch der gebildeten klingt th sehr oft nicht mehr als reine spirans. Wir dürfen darin nicht mit R. v. Raumer etwas altertumliches sehen, als ob das $t h$ jetzt erst im begriff wäre vollständig zur spirans zu werden, sondern die sache ist gerado umgekehrt: die spirans ist im begriff in den verschlusslaut uberzugehen. Ich glaube hinlänglich gezeigt zu haben, dass jene das ursprüngliche war, und es ist kaum noch nötig auf einige lautubergänge der ältern sprache aufmerksam zu machen, die für sie sprechen. Im nags. geht $h$ vor $t$ öfter in $b$ iiber (Koch 181), wofür sich auch $z$ geschrieben findet z. b. dozter (Koch 171), also wider der wechsel der : spiranten. Ferner geht $b$ in der 2. pers. sing., mitunter auch im pl. praes. in $s$ uiber; der anfang dazu findet sich bereits in den northumbrischen evangelien (Koch, flexionslehre 57). Das wichtigste argument aber ist wiler der eintritt der erweichung. Jetzt gibt es wol in dem grösseren teile von England kein anlautendes $t h$ mehr. Sowol in Schottland als in den südlichen 
dialekten wird dentales $d$ gesprochen, durch die artikulationsstelle von dem alten cacuminalen $d$ geschieden. Dass diess erst in neuerer zeit entstanden ist, beweist die ältere schrift. Die aussprache der gebildeten Englïnder ist die ubergangastufe dazu. Wie haben wir nun diese zu definieren? Nach R. v. Raumer und Scherer schwankte die aussprache zwischen spirans, verschlusslaut und aftricata. Ich muss mich gegen dieselben auf das zeugnis meines freundes Eduard Sievers berufen, der bei einem mehrmaligen längeren aufenthalt in England der aussprache die sorgfältigste aufmerksamkeit geschenkt hat, und dessen scharfe beobachtungsgabe in lautphysiologischen dingen ich reichlich bewährt gefunden habe. Nach ihm kann von einem doppellaute nicht die rede sein. Vielmehr besteht die zwischenstufe darin, dass die organe einander fast bis zu völligem verschluss genähert werden, und dass der laut möglichst kurz gesprochen wird, was in direktem widerspruch mit der natur eines doppellautes steht, der gerade eine verlängerung sein würde. Das ist gewissermassen eine mitte zwischen verschlusslaut und spirans, die leicht nach der einen, wie nach der andern hiniberschwankt, aber keine verbindung von beiden.

In derselben weise wie im englischen, haben wir auch im hoch - und niederdeutschen den ubergang des $t h$ in $d$ aufzufassen, nur dass die entwickelung hier rascher vor sich gegangen und weiter vorgedrungen ist. Dass schon im Heliand im inlaut erweichung eingetreten ist, erkennen wir daraus, dass uberwiegend $\delta$ geschrieben wird, was dann auch mit $d$ wechselt gerade wie im ags., so dass auch hier bald das eine, bald das andere als älter aufzufassen ist. Fur das alter der erweichung im fries., wo $\partial$ unbekannt ist und nur th geschrieben wird, sprechen die $d$ fur th in der lex Frisionum, die wol wie die gotischen aufzufassen sind. Im oberdeutschen und fränkischen zeigen schon die ältesten denkmäler die erweichung auch im anlaut. Isidor hat $d h$. Die ältesten alemannischen quellen haben zwar $t h$; da aber daneben $d$ schon uberwiegt, so können wir darin nur eine unvollkommene lautbezeichnung sehen, ebenso in dem th der jüngeren fränkischen quellen, das nicht erst wider rerhärtet sein kann und das auch unmittelbar in $d$ tibergeht, whne dass eine andere lautbezeichnung dazwischen läge. Für den Heliand ist es wahrscheinlich, wenn auch nicht zweifellos, 
dașs der anlaut noch nicht von der erweichung ergriffen ist, da hiẹr th durchsteht, während doch das zeichen $\partial$ bekannt ist. Zweifelhaft kann es auch sein, ob der auslaut mit stimmton gesprochen wurde oder nicht. Doch spricht die sonstige analogie fur das letztere, und die $\partial$ können hier eben so wenig tönende qualität beweisen wie die $\mathrm{t}$. Als entscheidend für anund auslaut zu gunsten der tonlosigkeit können wir es vielleicht betrachten, dass bei dem zusammenstoss eines auslautenden und eines anlautenden dentals th zuweilen in $t$ übergeht, so quatthat, quattiu, anttat aus quath that, quath thiu, and that, wider ein beispiel yon der abneigung gegen spiranten in consonantenverbindungen. Ueber die zeit, in welcher der ubergang zum verschlusslaute erfolgte vgl. Braune in diesem hefte s. $53 \mathrm{ff}$. Inlautend besteht auch heute die spirans, vielleicht von ältester zeit erhalten, in Holstein.

Ich habe mit möglichster vollständigkeit zusammenzustellen gesucht, was für natur und entwickelung der fraglichen laute in den verschiedenen dialekten von wichtigkeit ist. Vielleicht bin ich zu ausführlich darin gewesen, da die meisten der von mir vorgebrachten tatsachen auch schon sonst allgemein bekannt sind. Da ich aber weiss, wie sehr man sich vielfach gegen die anerkennung gewisser lautübergänge sträubt, so schien es mir nötig dieselben so sicher als möglich zu begründen. Vor allem kam es mir darauf an durch cine zusammenhängende darstellung aller in betracht kommenden einzelheiten die ubereinstimmung in denselben klar hervortreten zu lassen. Diese zusammenfassende und vergleichende betrachtung muss unser urteil bestimmen, wo die bei den einzelheiten stehenbleibende untersuchung hie und da noch zweifel zurücklässt. Ueberblicken wir die gewonnenen resultate, so ergibt sich als urgermanischer lautstand folgendes: Die indogermanischen tenues sind zu tonlosen spiranten geworden $h, b, f$. Die indogermanischen aspiraten sind inlautend zu tönenden spiranten geworden; nur nach nasalen erscheinen sie, soweit wir sie zurückverfolgen könncn, als tönende verschlusslaute. Diese ausnahme begreift sich leicht; denn da die nasale durch verschluss des mundcanals gebildet werden, so schliesst sich daran ein explosivlaut leichter an, als ein reibelaut, zu dem erst, bevor er gebildet werden kann, der verschluss gelockert werden muss. Ebenso 
zeigt sich im anlaut von alter zeit an tönender verschlusslaut. Jedoch muss im ältesten niederdeutschen und angelsächsischen wenigstens zum teil gutturale spirans bestanden haben. Es ist ferner nicht ganz sicher, ob schon zur zeit des Ulfilas $b, g, d$ im anlaut wirkliche verschlusslaute waren oder zwischen verschlusslaut und spirans schwankten. Das ist der boden, auf dem die weitere entwicklung ruht.

Es zeigt sich nun, lass eine zwiefache bewegung der ursprünglichen indogermanischen verschlusslaute zum teil zwischen die erste, die gemeingermanische und die zweite, die specifisch hoch(leutsche verschiebung, *) zum teil in die letztere hinein und nach ihr fällt, die erweichung der aus indogermanischer tenuis entstandenen spiranten, besonders im inlaut, und der tibergang der tönenden, in einem falle auch der tonlosen spiranten in verschlusslaute. Was die erstere betrifft, so beginnt sie vor der auf uns gekommenen überlieferung und tritt zunächst sporadisch auf, zum grösseren teil ubereinstimmend in den verschiedenen dialekten, was aber eine reihe von einzelnen abweichungen nicht ausschliesst. Das verhältnis ist sehr ähnlich wie bei dem tibergange ron $a$ zu $e$ in den versehiedenen europäischen sprachen. Bei dem weiterumsichgreifen der erweichung scheiden sich die dialekte etwas mehr, so dass aber doch die entwickelung in den einzelnen sehr analog ist. $f$ wird inlautend allgemein tönend ausser im hochdeutschen und einem teile des mitteldeutschen, wo sich einige tonlose $f$ erhalten, im nordischen auch auslautend. Der ubergang des $h$ zu $g$ bleibt sporadisch, jedenfalls nur deshalb, weil das $h$ sehr frih zum blossen hauch wurde, welcher durch den stimmton nur zum spiritus lenis werden konnte. Wir haben daher das schwinden des $h$ im inlaut wischen rokalen, welches ausser im oberdeutschen

*) Die namen „erste und zweite verschiebung“ wären vielleicht besser ganz zu vermeiden; sie haben wenigstens nur einen praktischen wert. In wirklichkeit sind nicht die sogenannten beiden verschiebungen je ein aus dem kreise aller ibrigen lautveränderungen heraustretendes ganze, sondern sie bilden mit den von uns besprochenen vorgängen, von denen man einen teil zur zweiten verschiebung zu rechnen pflegt, und mit andern erst später eintretenden veränderungen eine reihe von vielen einzelnen lautwandelungen, dic sich von der ältesten zeit bis auf die neueste nach einander und meist ohne beziehung zu einander vollziehen. 
allgemein erfolgt; wol als eine analoge erscheinumg anzusehen. Die erweichung des $b$ ist inlautend allgemein (im nordischen und englischen findet sie auch auslautend statt), erstreckt sich aber in Deutschland durchgängig, in England und Skandinavien partiell auch über den anlaut. Die neigung zur erweichung ist also bei den dentalen am stärksten, aber die behandlung ist keine grundverschiedene. Unsere auffassung erhält dadurch noch eine schlagende bestätigung, dass sich in völlig analoger weise wie die erst auf germanischem boden entstandenen spiranten, auch die einzige indogermanische tonlose spirans $s$ entwickelt. Auch hier ist die erweichung zuerst sporadisch, im got. durch $z$ bezeichent, und die in ältester zeit erweichten $s$ gehen in $r$ tiber, ebenfalls im ganzen ubereinstimmend, aber doch mit mannigfachen abweichungen in den einzelnen dialekten. Nach der periode des rotacismus geht dann die erweichung weiter, wird im anlaut ausser in Oberdeutschland allgemein, ausgenommen in der gemination und in verbindung mit tonlosen consonanten, und ergreift im niederdeutschen und einem teile des mitteldeutschen, auch in englischen dialekten selbst den anlaut. Der letztere umstand ist besonders wichtig in rücksicht auf die erweichung des $b$. Englische dialekte kennen auch die erweichung des $f$ im anlaut. Bei allen harten spiranten zeigt sich also dieselbe tendenz. Die vollkommenste analogie dazu findet sich im lateinischen. Ascoli (Vergleichende lautlehre 171. ff.) hat meiner ansicht nach uberzeugend nachgewiesen, dass die lateinischen inlautenden medien $=$ indogermanis̀chen aspiraten aus den.ursprtunglich wie im griechischen verhärteten und dann zu tonlosen spiranten gewordenen aspiraten erweicht sind. Unsere auffassung der deutschen und Ascolis der lateinischen lautwandelungen stützen sich gegenseitig. Ebenso werden im altirischen die aus indogermanischer tenuis entstandenen sogenanten aspiraten, die aber sicher als spiranten aufzufassen sind, inlautend erweicht; daher das schwanken in der schreibung zwischen aspirata und media. Wir haben hier also noch eine genauere ubereinstimmung mit dem deutschen.

Die zweite bewegung, der übergang in den verschlusslaut, hat statt sowol bei den aus den medienaspiraten entstandenen, von anfang an tönenden spiranten, als bei den aus den ton- 
losen erweichten und den ursprünglichen indogermanischen weichen spiranten $(j$ und $w)$, nur in verschiedener ausdehnung. Am regelmässigsten und fast durchgängig tritt sie ein nach nasalen und und in dor gemination, dann unter dem einflusse anderer vorhergehender, zum teil auch unter dem folgender consonanten. Aber auch ohne einen solchen einfluss erfolgt sic zwischen vokalen, ziemlich allgemein im oberdeutschen, bei den dentalen auch im niederdeutschen und (wenigstens bei den ursprünglich tönenden oder in ältester zeit tönend gewordenen) angelsächsischen, bei den gutturalen auch im schwedischen, vereinzelt noch sonst. Bei den dentalen geht also auch diese bewegung am weitesten und ergreift selbst den anlaut, da auch dieser von der erweichung betroffen wird. Darin liegt indessen kein wesentlicher unterschied. Eine grössere abweichung besteht darin, dass im dänischen und schwedischen der hart gebliebene spirant $b$ gleichfalls zum verschlusslaut wird, was bei dem gutturalen und labialen spiranten nicht möglich war, weil $h$ zum blossen hauch, $f$ labiodental geworden war. Wol findet hie und da auch eine entgegengesetzte bewegung statt. So geht im dänischen die aus der tenuis erweichte media in die spirans über. Im ags. und engl., sowic im alts. finden sich schwankungen des $d$ nach $\partial$ und th. Aber der allgemeine zug ist vom reibelaut zum verschlusslaut, und ersterer wird, so lange nicht das gegenteil erwiesen ist, immer zunächst für älter gelten mitissen. Widerun ist die entwickelung im lateinischen analog: die inlautend erweichten spiranten werden zu tönenden verschlusslauten.

Ganz sichere beispiele vom tibergang des dentalen spiranten in den verschlusslaut haben wir im persischen, wo $z:=\mathrm{skr}$. $h$ zu $d$ wird. Wenn in neugriechischen dialekten $\vartheta \mathrm{zu} \tau, \chi \mathrm{zu} x$ wird (rgl. Mullach, Grammatik der griechischen vulgärsprache s. 28. 89. 94. Curtius, Grundz. 3. s. 386. Ascoli, Vergleichende lautlehre s. $161 \mathrm{ff}$.), so ist es im höchsten grade unwahrscheinlich, dass in diesen füllen die aspirata ganz abweichend von der sonstigen entwickelung niemals zur spirans geworden sein, sondern nur den hauch eingebtisst haben sollte. Vielmehr werden wir uns den vorgang zu denken haben wie im schwedischen und dänischen. Den übergang in den verschlusslaut haben wir uns wol überall so vorzustellen, wie ihn uns die heutige 
aussprache des englischen th lehrt und wie er bei besprechung desselben erörtert ist. Auch im neugricehischen soll die aussprache des $\vartheta$ zwischen reibe- und verschlusslaut schwanken. Auch hier wird es falsch sein, wenn man es fuir einen doppellaut erklärt, oder wenn man meint, dass diesc aussprache altertumlich sei und der laut erst im begriff in die blosse spirans iberzugehen. Vielmehr ist er im übergange aus der spirans in den verschlusslaut begriffen und teilweise ist dieser thergang schon vollzogen.

Im anlaut haben, soweit unsere zeugnisse zurickgehen, die len indogermanischen medienaspiraten entsprechenden laute eine andere gestalt als im inlaut. Doch bleibt es immer unsicher, ob schon im gotischen wirkliche explosivlaute bestanden, und im altniederdeutschen und angelsächsischen ist sicher zum teil gutturaler spirant das älteste. Es fragt sich nun, in welcher weise diese zweiheit auf die unzweifelhaft friber bestehende einheit zurückzufuhren ist. Ist die spirans aus dem verschlusslaute oder der verschlusslaut aus der spirans oder beide unabhängig aus einem dritten laute entstanden? Ersteres war bisher die allgemeine annalıme, indem man den verschlusslaut vielfach da ansetzte, wo wir die spirans erkannt haben, und indem man die mit recht oder mit unrecht im inlaut angesetzten verschlusslaute für ursprtinglich hielt, während wir ihre entstehung aus den reibelauten nachgewiesen haben. Somit ist dieser ansicht der boden entzogen. Es ist gar keine veranlassung anzunehmen, dass die spirans erst aus dem verschlusslaute entstanden sei, um damn wider teilweise in denselben uberzugehen. Viel grössere wahrscheinlichkeit hat die zweitc möglichkeit, die ontstehung des verschlusslautes aus der spirans. Sie stimmt durchaus zu dem grundzuge der dargestellten entwickelung. Was ist wahrscheinlicher, als dass derselbe zug, welcher einen langen zeitraum. bindurch die tönencle spirans im inlaut und, soweit gelegenheit dazu war, auch im anlaut zum verschlusslaut hindrängte, auch schon eine kurze zeit vor der periode wirksam gewesen ist, aus der unsere ältesten denkmäler stammen? Ja er muss es sogar, da er bereits in gotischen inlautend nach $n, r, l, z$ unzweifelhaft gerwirkt hat. Gleichzeitig mit dem gemeingermanischen übergange nach nasalen kann auch der im anlaut stattgefunden haben. Wir sehen auch 
sonst in der deutschen lautgeschichte den inlaut nach consonanten in gleicher weise behandelt wie den anlaut. Dass der anlaut den verschlusslaut mehr liebt, als der inlaut, besonders zwischen vokalen, zeigen das englische, schwedische und dänische. Die dritte möglichkeit ist noch ins auge zu fassen, ob etwa die entwickelung der indogermanischen laute von anfang an oder von einer bestimmten noch vor der einfachen spirans. und dem einfachen verschlusslaute liegenden tubergangsstufe an fuir den an- und inlaut verschiedene wege eingeschlagen hat. Diess führt uns aber auf die frage nach der ursprunglichen beschaffenheit der zu grunde liegenden laute in der indogermanischen ursprache.

Hierüber sind nun drei*) verschiedene ansichten aufgestellt. Die eine behauptet wirkliche aspiraten, die zweite affricaten, die dritte einfache spiranten. Die letzte ist jetzt wol allgemein aufgegeben. Der streit dreht sich noch um die erste uberwiegend anerkannte und die zweite von R. v. Raumer und Scherer vertretene. Es würde uns zu weit führen alles, was zu gunsten der einen oder andern ansicht vorgebracht ist, hier noeh einmal zu widerholen. Ich halte dafür, dass der beweis für die ursprünglichkeit der aspiraten im sanskrit und griechischen geliefert ist durch Curtius, Grundzitge ${ }^{3} 383$ ff. und Ascoli, Vergleichende lautlehre $149 \mathrm{ff}$., wenn ich auch einige der von ihnen vorgebrachten argumente nicht gelten lassen kann. Die auffassung der laute als spiranten ist auch von R. v. Raumer sehr grindlich widerlegt, Sprachwissenschaftliche sehriften $96 \mathrm{ff} .383 \mathrm{ff}$. Gegen die affricaten entscheidet meiner uberzeugung nach die metrik des griechischen und des sanskrit. Wenigstens kann ich mir noch keine vorstellung von den Raumerschen affricaten machen, die keine positionmachende doppellaute sein sollen. Scherer (zeitschr. f. ll. östr. gymn. XII, 641) hält durch den übergang von $t v a$ in $t^{t} a$, den er als assimilation des organes auffasst $\left(t^{b} a=t s a\right)$, die affricaten im sanskrit fur bewiesen. Aber um eines solchen einzelnen lautiberganges willen, für den eine andere auffassung sehr wol denkbar ist, kann man nicht

*) Von dem streite iiber die ursprüngliche tonbegabtheit oder tonlosigkeit dieser laute, welche letztere noch in neuerer zeit durch Rud. v. Raumer vertreten wird, kann ich für unsern zweck absehen. 
alle entgegenstehenden argumente ignorieren. Die verwandelung einer verbindung von zwei homorganen tönenden lauten in eine von zwei durch das organ wie durch den stimmton verschiedenen lauten bleibt eine unlösbare physiologische schwierigkeit, während der entgegengesetzte vorgang leicht begreiflich und durch reichliche analogieen gestützt ist. Wir wollen trotzdem auf alle drei aufgestellten ansichten rücksicht nehmen und sehen, wie sich nach einer jeden unsere frage stellt. Wären die indogermanischen laute spiranten gewesen, so wäre die frage einfach entschieden. Es wäre dann selbstrerständlich nur die zweite von uns angesetzte möglichkeit anzunehmen. Die spiranten wären in germanischen inlautend zunächst geblieben, anlautend in verschlusslaute übergegangen. Schwieriger ist es, wenn, was unzweifelhaft der fall ist, eine von den beiden andern ansichten richtig ist, über den gang der entwickelung zu entscheiden. Die vertreter beider nehnen übereinstimmend an, dass das hinter dem verschlusslaut stehende element abgefallen sei, der hauch oder die homorgane spirans, und so die media entstanden. Man beruft sich dabei, Scherer allerdings nicht, auf die tibereinstimmung der iranischen, slavischen, litauischen und keltischen sprachen, für die man die gleiche entwickelung annimmt. Namentlich wird der übergang im slavischen und litauischen mit dem im germanischen in historischen zusammenhang gesetzt, und man sieht darin den beginn der lautverschiebung, der noch in die periode der slavodeutschen spracheinheit fallen soll. Ich stimme mit Scherer darin tiberein, dass dann notwendig die alte und die neue media im germanischen, wie im slavischen, zusammengefallen sein müsten. Es lässt sich hierbei auch nicht wol eine zwischenstufe denken, bis zu welcher etwa nur das deutsche gemeinsam mit dem slavischen gegangen wäre. Der hauch war entweder weg oder noch da. Eine andere art von zwischenstufe ist noch angenommen, nämlich, dass zwar der hauch im slavodeutschen verschwunden, zunächst aber geflüsterte media entstanden sei, die dann unabhängig im slavischen und deutschen, im letzteren erst nach der verschiebung der media zur tenuis, tönend geworden sei. Aber der weg, den die media zur tenuis nalım, ging notwendig durch die geflusterte media hindurch, und es muste so zusammenfall eintreten. Wurde die medien-aspirata oder affricata zur 
media durch abfall des zweiten elements, so kaun diess erst nach der verschiebung der media zur tenuis, also auch erst nach der verschiebung der ursprïunglichen tenuis*) auf speciell germanischem boden geschehen sein, wie auch Scherer annimmt; man darf sich also nicht zur stütze dieser ansicht auf die tibrigen sprachen berufen. ${ }^{* * *}$ ) Wir werden also ein recht haben lediglich die älteste lautgestaltung des germanischen mit der des indogermanischen zu vergleichen und zu sehen, auf welche weise sich dieselben am einfachsten vermitteln. Wir haben gesehen, dass wir im germanischen für den inlaut auf die spirans als das älteste kommen, und dass es unwahrscheinlich ist, dass dieselbe aus der media entstanden ist. Ein sehr häufiger lautübergang ist die entstehung tonloser spirans aus tonloser affricata oder aspirata durch die affiricata hindurch. Danach ist es auch die nächstliegende annahme, dass die germanischen tönenden spiranten aus den medienaspiraten durch die medienaffricaten hinduich, oder, wenn man doch letztere für ursprünglich halten wollte, noch einfacher aus diesen entstanden sind. Für den anlaut könnte man nun bei der alten theorie von dem abfall des hauches oder spiranten stehen bleiben. Dann hätten wir also von vornherein oder von der zwischenstufe der medienaffiricaten an verschiedene entwickelung des an- und inlautes. In dieser fassung ist die lehre von der verschicbung der aspiraten von E. Sievers bereits in seiner im wintersemester 71/72 gehaltenen vorlesung uber deutsche grammatik vorgetragen.

*) Fuir die prioritït der verschiebung der tenuis vor der der media liesse sich vielleicht ausser dem von Scherer gegebenen beweis, gegen den meiner über\%eugung nach kein widerspruch möglich ist, die gotische nanensform Kreks = Gracus anfiihren, die auch dem althochdeutschen kriach zu grunde liegt. Sie wiirde sich vielleicht so erklären, dass das wort in einer zeit entlehnt wurde, als die verschiebung der tenuis bereits vollzogen war, die der media noch nicht, so dass es nur noch von der letzteren betroffen wurde.

**) Man miiste dann etwa annehmen, dass die lautverschiebung in eine zeit zuriickreicht, in welcher das deutsche noch ein dialekt der indogermanischen ursprache war, der mit den ibrigen dialekten in ununterbrochenem zusammenhange stand. Ich glaube nicht, dass jemand diess wahrscheinlich finden wird. Dass das in der vorigen anmerkung angetiihrte argument dagegen streitet, wage ich nicht geltend zu machen, da es doch nur eine unsichere vermutung bleibt. 
Es lässt sich indessen zunächst kein grund einsehen, warum in- und anlaut ganz verschiedene wege eingeschlagen haben sollten. Dann aber scheint mir der gewöhnlich als etwas so leichtes angesehene abfall des hauches oder der homorganen spirans von physiologischer seite höchst bedenklich. Es ist häufig, dass hauchlose tenuis aspiriert wird, aber einmal vorhandene aspiration pflegt nicht spurlos zu verschwinden. Die aus dem nordischen und dem neugriechischen beigebrachten beispiele vom verlust des hauches in harten aspiraten sind, wie wir gesehen haben, anders zu fassen. Verändern sich die aspiraten so gehen sie in affricata und dann in spirans über. Noch weniger hat der abfall der spirans in der affricata irgend welche wahrscheinlichkeit. Wir haben gesehen, dass dic dafür namentlich aus dem englischen angeführten beweise nicht zutreffen. Ein solcher abfall müste wie jeder ausfall eines consonanten in einer consonantenverbindung auf assimilation beruhen. Es ist aber ein allgemeines gesetz, dass in der regel der zweite laut tiber den ersten den sieg davonträgt, und ein noch allgemeineres, dass die dauerlaute ein entschiedenes übergewicht über die momentanen behaupten. Daher die verwandlung der affricata zur spirans. Nehmen wir also an, dass sich die aspiraten im anlaut zunächst in gleicher weise wie im inlaut zu spiranten entwickelten und daraus erst zu tönenden verschlusslauten. Das stimmt völlig zu dem, was wir bloss vom standpunkte des germanischen aus als wahrscheinlich erkannt haben.

Ist diess die richtige auftassung der entwickelung, so wird die von Scherer aufgestellte reihenfolge der verschiebung (tenuis media, aspirata oder affiricata) wider zweifelhaft. Die verschiebung der aspirata zur spirans konnte hinter, zwischen und vor die beiden andern verschiebungen fallen, ohne dass eine vermischung eintrat. Nur muss der übergang der spirans in die media nach der verschiebung der alten media erfolgt sein. Es wäre also ins auge zu fassen, ob nicht doch grüude vorhanden sind; die uns bestimmen die verschiebung mit den aspiraten beginnen zu lassen und ob dann nicht doch ein historischer zusammenhang mit den verwạnten sprachen besteht. Wir betreten hier allerdings ein gebiet, auf dem sich kaum etwas anderes aufstellen lässt, als mehr oder minder wahrscheinliche hypothesen. Folgendes, worauf ich von herrn professor Leskien auf- 
merksam gemacht worlen lin, liesse sich vielleicht für die prioritait der verschicbung der aspiraten geltend machen. Das $t$ in den praeteritis mahta, ohla und dem im got. nicht tiberlieferten, aber vorauszusetzenden dauhta kann nicht wie in thahta, aihta, mosta etc. durch assimilation an den voraufgehenden consonanten entstanden scin. Man könnte sich nun den vorgang so denken: durch die rerschiebung der aspiraten zur spirans

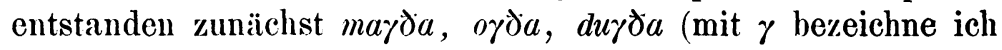
die gutturule tönende spirans); da aber eine spirans hinter einer anderen die neigung hatte in den verschlusslaut überzugehen, wie wir auch an den praeteritis thahta etc. sehen können, und wie es sich auch in den späteren germanischen dialekten, namentlich im altn. zeigt, so erfolyte hier dieser ubergang vielleicht alsbald vor der verschiebung der alten media und das neue $d$ wurde damn durch denselben akt wie diese zur tenuis verschoben. Für vollkommen sicher will ich diese erklärung nicht ausgeben, da sich in den praeteritis noch andere unregelmässigkeiten zeigen \%. b. kunpa.

Ein zusammenhang mit den verwanten sprachen, welche die aspirata zur media cntwickelt haben, der allerdings von vornherein schr wahrscheinlich ist, war bei der bisherigen auffassung der verschiebung unmöglich. Durch unsere aufstellung gewinnen wir zunächst die möglichkeit die verwandelung der aspiraten vor die audern verschiebungsakte zu stellen. Wenn aber cin zusammenhang mit den ubrigen sprachen bestehen sollte, so niuste auch der gang der verschiebung in diesen derselbe wic im germanischen gewesen sein. Es fragt sich, ob dies möglich oder vielleicht wahrscheinlich ist. Wir haben gesehen wie mislich es mit der erklärung der media aus abfall des hauches oder des homorganen spiranten bestellt ist. Die vielfach angezweifelte möglichkeit des überganges aus der sp1rans in den verschlusslaut glaube ich durch meine untersuchung festgestellt zu haben. Der einzige weg einen historischen zusammenhang oder auch nur eine analogie des überganges zwischen dem germanischen und den iibrigen sprachen herzustellen, bleibt die anuahne, dass in ihnen allen die aspirata zunächst zur spirans und dann zur media geworden sei.

Es wäre nun zu untersuchen, ob sich für diese annahme noch aus den einzelnen sprachen grtinde beibringen liessen. Im 
Zend stehen anlautend $h, g, d$, inlautend $b h, g h$, $d h$, welche letzteren gewöhnlich als spiranten aufgefasst werden. Allerdings hat Ascoli in den Studj Irani, articolo primo (vgl. Kuhns zeitschr. 17, 135 ffi.) den nachweis zu führen gesucht, dass man im Zend wirkliche tonlose und tönende aspiraten anzunehmen habe. Soweit ich darüber urteilen kann, halte ich durch Ascolis ausführungen die ältere ansicht noch nicht für widerlegt. Namentlich wird die entstehung von tönender aspirata aus alter media, wie sie Ascoli annehmen muss, da auch diese im inlaut durch $b h, g h$, $d h$ vertreten wird, eine physiologische unmöglichkeit bleiben. Sind $b h, g h, d h$ spiranten, so haben wir ein ganz ähnliches verhältnis wie im deutschen. Nur freilich lässt sich die ursprünglichkeit dieser spiranten nicht erweisen weil sie auch für die alte media stehen, also auch, wo sie die aspirata vertreten, erst aus der media entstanden sein können. Doch kann man auch nicht mit sicherheit behaupten, dass letzteres der fall ist. Es ist leicht möglich, dass durch dieselben ursachen die spirans im inlaut erhalten blieb, durch welche die media in sie verwandelt wurde, ja sogar, dass der wechsel zwischen verschluss- und reibelaut, der bei der alten a spirata bestand, auch auf die alte media einwirkte, und so eine ausgleichung eintrat. Dasselbe verhältnis haben wir im dänischen, wo im inlaut die aus der tenuis erweichten medien $\mathrm{zu}$ spiranten werden. Aehnlich ist auch der wechsel von $o$ und $u$ im germanischen, die sich in ganz analoger weise verhalten, mag. $a$ oder $u$ der grundvokal und demnach $o$ oder $u$ das ältere sein. Wir können also aus dem Zend nichts mit sicherheit fur noch gegen unsere hypothese beibringen.

Ebenso lässt uns das keltische in ungewisheit. Auch im altirischen stehen im inlaut tönende spiranten, gewöhnlich als aspiraten bezeichnet. Es unterliegt aber wol keinem zweifel dass die irischen aspiraten als spiranten zu fassen sind. In der ältesten zeit wird allerdings noch die einfache media geschrieben; aber ihr wechsel mit den harten aspiraten (spiranten) macht es wahrscheinlich, dass wir darin nur eine mangelhafte orthographie zu sehen haben. Aber ebenso wie im Zend ist die alte media gleichfalls zur spirans geworden und ganz mit der alten aspirata zusammengefallen.

Im slavischen und litauischen steht tiberall die media, nir- 
gends zeigt sich die spirans. Ihr einstiges vorhandensein aber glaube ich mit einiger wahrscheinlichkeit aus einem sonst kaum begreiflichen lautübergange schliessen zu können. Es ist bekannt, dass in dem zur casusbildung verwanten suffixe bhi das bh ubereinstimmend im slavischen, litauischen und deutschen, soweit die betreffenden casus erhalten sind, in $m$ tibergangen ist. Der übergang ist von so besonderer art, dass er notwendig in die periode des ungetrennten zusammenlebens der drei sprachfamilien fallen muss. Ebenso ist es klar, dass die zunächst vorhergehende lautgestaltung in allen dreien dieselbe gewesen ist. Ein unmittelbarer übergang von $b h$ in $m$ ist undenkbar, der von $b$ in $m$ nicht wahrscheinlich, ausserdem kann aber ein $b$, wie wir gesehen haben, in der slavodeutschen periode nicht bestanden haben. Nicht selten dagegen in den verschiedensten sprachen ist der wechsel von reinlabialer spirans mit $m$. Diese werden wir als die notwendige zwischenstufe zwischen $b h$ und $m$ betrachten. Nun ist es aber durchaus nicht wahrscheinlich, dass $b h$ nur in diesem falle in die spirans ubbergegangen sein sollte, während es sich sonst ganz anders entwickelt hättẹ. Alles stimmt vortrefflich, wenn unsere hypothese richtig ist.

Eine hypothese kann allerdings unsere ansicht nur genannt werden; aber man wird ihr mindestens die selbe wahrscheinlichkeit zugestehen müssen, als der hypothese vom abfall des hauches, die für etwas unbestreitbar feststehendes ausgegeben wird. Wir würden also nach unserer auffassung fur das iranische, slavische, litauische, germanische und keltische als das zunächst zu grunde liegende gemeinsame die tönende spirans anzusehen haben. Vom standpunkte dieser sprachen aus könnten wir nicht weiter gelangen, und es stände nichts in wege darin das ursprüngliche zu sehen. Aus ihnen lässt sich auch kein argument gegen die ursprunglichkeit der medienaffiricaten beibringen, wenn sie auch eben so wenig durch sie gefordert wird. Die entscheidung ist aus dem sanskrit und griechischen herzuholen und, insofern die verhärtung der italischen spiranten mit der der griechischen aspiraten in zusammenhange steht und richtig aus assimilation an das tonlose $h$ erklärt wird, indirekt auch aus den italischen sprachen.

LEIPZIG.

Beiträge zur geschichte der deutschen sprache. I.

\section{HERMANN PAUL.}

